\title{
The molecular interplay of the establishment of an infection - gene expression of Diaphorina citri gut and Candidatus Liberibacter asiaticus
}

Flavia Moura Manoel Bento ${ }^{1}$ (D) Josiane Cecília Darolt ${ }^{2,3}$ (D), Bruna Laís Merlin ${ }^{1}$ (D) Leandro Penã ${ }^{2,4}$ (D), Nelson Arno Wulff',3 (D) and Fernando Luis Cônsoli ${ }^{1 *}$

\begin{abstract}
Background: Candidatus Liberibacter asiaticus (CLas) is one the causative agents of greening disease in citrus, an unccurable, devastating disease of citrus worldwide. CLas is vectored by Diaphorina citri, and the understanding of the molecular interplay between vector and pathogen will provide additional basis for the development and implementation of successful management strategies. We focused in the molecular interplay occurring in the gut of the vector, a major barrier for CLas invasion and colonization.

Results: We investigated the differential expression of vector and CLas genes by analyzing a de novo reference metatranscriptome of the gut of adult psyllids fed of CLas-infected and healthy citrus plants for 1-2, 3-4 and 5-6 days. CLas regulates the immune response of the vector affecting the production of reactive species of oxygen and nitrogen, and the production of antimicrobial peptides. Moreover, CLas overexpressed peroxiredoxin, probably in a protective manner. The major transcript involved in immune expression was related to melanization, a CLIP-domain serine protease we believe participates in the wounding of epithelial cells damaged during infection, which is supported by the down-regulation of pangolin. We also detected that CLas modulates the gut peristalsis of psyllids through the down-regulation of titin, reducing the elimination of CLas with faeces. The up-regulation of the neuromodulator arylalkylamine $\mathrm{N}$-acetyltransferase implies CLas also interferes with the double brain-gut communication circuitry of the vector. CLas colonizes the gut by expressing two Type IVb pilin flp genes and several chaperones that can also function as adhesins. We hypothesized biofilm formation occurs by the expression of the cold shock protein of CLas.
\end{abstract}

\footnotetext{
* Correspondence: fconsoli@usp.br

${ }^{1}$ Insect Interactions Laboratory, Department of Entomology and Acarology,

Luiz de Queiroz College of Agriculture, University of São Paulo, Avenida

Pádua Dias 11, Piracicaba, São Paulo 13418-900, Brazil

Full list of author information is available at the end of the article
}

(c) The Author(s). 2021 Open Access This article is licensed under a Creative Commons Attribution 4.0 International License, which permits use, sharing, adaptation, distribution and reproduction in any medium or format, as long as you give appropriate credit to the original author(s) and the source, provide a link to the Creative Commons licence, and indicate if changes were made. The images or other third party material in this article are included in the article's Creative Commons licence, unless indicated otherwise in a credit line to the material. If material is not included in the article's Creative Commons licence and your intended use is not permitted by statutory regulation or exceeds the permitted use, you will need to obtain permission directly from the copyright holder. To view a copy of this licence, visit http://creativecommons.org/licenses/by/4.0/ The Creative Commons Public Domain Dedication waiver (http://creativecommons.org/publicdomain/zero/1.0/) applies to the data made available in this article, unless otherwise stated in a credit line to the data. 
Conclusions: The thorough detailed analysis of the transcritome of $C$. L. asiaticus and of D. citri at different time points of their interaction in the gut tissues of the host led to the identification of several host genes targeted for regulation by L. asiaticus, but also bacterial genes coding for potential effector proteins. The identified targets and effector proteins are potential targets for the development of new management strategies directed to interfere with the successful utilization of the psyllid vector by this pathogen.

Keywords: Host regulation, Effector proteins, Host - pathogen interactions, Microbial ecology, Infection strategies, Host immunity

\section{Background}

Bacterial plant pathogens are adapted to infect and propagate in insect tissues, as many insects are used as vectors by plant pathogens to infect new host plants and spread themselves in the environment. Plant pathogenic bacteria vectored by insects that use persistent, circulative, propagative mode of transmission require plastic phenotypes to interact with the different enviroments represented by sieves and tissues of the host plant, and the diverse environments faced in the gut lumen, hemocele and different tissues of the vector insect during the processes of acquisition and vector competence development [1]. The capability of these pathogens to infect completely different hosts (plant and insect), and yet to depend on the shuttle host (insect) to locate and collect the pathogen in a diseased plant, and later transport it to new, health host plants for the establishment of new infections requires a set of strategies devoted to manipulate the host insect $[2,3]$. The understanding of the interactions pathogens and hosts have at their molecular level during the processes of host invasion and infection establishment is required for a fully comprehension of the mechanisms involved in guaranteeing the host pathogen association. Such mechanisms of close interactions in the associations of host and pathogens represent potential new targets for the development of new technologies and/or use of existing technologies to interfere with the successful association pathogens establish with their hosts.

Candidatus Liberibacter are bacteria posing serious threat to food security worldwide by causing diseases to potatoes and citrus. Citrus is certainly the most severely damaged crop by $\mathrm{Ca}$. Liberibacter infections, as three different species are known to infect citrus plants $(\mathrm{Ca}$. L. asiaticus, $C a$. L. americanus and $C a$. L. africanus), causing the incurable greening or huanglongbing (HLB) disease [4-7]. Candidatus Liberibacter did not make to the top 10 plant pathogenic bacteria [8], but HLB has caused a tremendous impact in the citrus industry and led to the complete elimination of citrus orchards and the interruption of citrus production in what used to be highly productive citrus centers $[7,9,10]$.

Candidatus Liberibacter asiaticus (CLas) is the most spread in the world, currently infecting citrus in the major producing areas [11-13]. In plants, Candidatus Liberibacter reside exclusively in the phloem sieve tubes $[4,14-16]$, but it will infect several tissues of their host vector insect [17-20]. CLas is vectored by the worldwidely distributed Asian Citrus Psyllid (ACP) Diaphorina citri Kuwayama (Hemiptera: Psyllidae) [4].

Despite the importance of this disease to the worldwide citriculture and all of the investments and efforts of the scientific community to understand the interactions of the pathogen with its vector, much of the mechanisms involved in the pathogen-vector interplay remain unknown. We learned that CLas is transmitted from host plant to host plant by establishing a persistent, propagative transmission mode with its vector, a transmission mode that is very common to plant viruses vectored by aphids and whiteflies [19, 21, 22]. CLas was also demonstrated to alter the host plant transcriptional profile [2325]. We also learned CLas infects salivary glands and the midgut of vectors, tissues that are often recognized as natural barriers to circulative, propagative pathogens as they can prevent translocation of pathogens within the vector host [17, 22, 26, 27]. Differential gene expression analysis of CLas-infected psyllids also demonstrated CLas alters the gene expression profile of of the gut, salivary glands and whole-body of D. citri [19, 28-30]. More recently, new information on the higher efficiency of adults than psyllid nymphs as vectors of CLas has been reported, with adults displaying a higher successful rate of infection of healthy citrus plants than nymphs [27]. Nevertheless, little information at the physiological and molecular level on the interface of the interactions of CLas with key vector tissues is available [31].

The limitations in the availability of efficient, costeffective strategies for the management of the vector and the disease prompted a large number of investigations for the exploitation of new technologies, and promising results were mainly obtained with RNAi-based approaches [32-35].

In here we focused on investigating the metatranscriptome of the gut of adults of D. citri feeding on CLasinfected citrus plants for different periods of time in order to identify genes of CLas that are expressed during the colonization of the gut of adults and the differential gene expression in the gut of psyllids over time against 
psyllids feeding on healthy, CLas-free citrus plants. Our major goal was to understand the dynamics of CLas gene expression during psyllid colonization and the response mechanisms that were activated in the gut epithelium of psyllids when exposed to $\mathrm{Ca}$. Liberibacter asiaticus cells. We believe our data represents a source of very specific targets for the development and implementation of new strategies of psyllid/disease control using RNAi and/or gene editing technologies such as the CRISPR-Cas9 system.

\section{Results}

\section{De novo transcriptome assembly}

Sequencing from libraries of the gut of uninfected and CLas-infected nymphs and adults of $D$. citri yielded $395,151,161$ reads, with an average of $16,464,632$ reads/ library. The use of the resulting 385,677,949 trimmed, quality-filtered reads (average 16,069,915 reads/library) allowed the de novo assembly of a transcriptome with 260,612,776 nucleotides (260 Mb), with transcripts with an average size of $481 \mathrm{bp}$ and a N50 of 2,095 bp. The assembly resulted in 248,850 transcripts with an average of $39.9 \%$ GC content. Annotation of the transcriptome allowed the putative identification of 90,531 transcripts (36.4\%), of which 52,081 transcripts were allocated to different gene ontology categories (Additional file 1). Additional filtering using the highest score hit after BlastX allowed the identification of 66,993 transcripts belonging to $D$. citri, 807 belonging to $C a$. Liberibacter, and 1,967 to the secondary symbiont Wolbachia, among others (Additional file 2).

\section{CLas gene expression in the gut of adults of Diaphorina citri}

Since we could not detect relevant read counts against transcripts belonging to $C a$. L. asiaticus when using samples obtained from nymphs, only samples collected at the adult stage were subjected to differential expression analysis. Gene expression analysis recognized 807 transcripts belonging to $\mathrm{Ca}$. L. asiaticus in the gut of adult psyllids fed on CLas-infected citrus plants in at least one of feeding exposure periods analyzed for adults (1-2 d; 3-4 d; 5-6 d - A1CLas ${ }^{+}$, A2CLas $^{+}$and A3CLas ${ }^{+}$, respectively), with no CLas-related transcripts being identified in gut samples from insects fed on healthy plants $\left(\mathrm{CLas}^{-}\right)$.

The detection of gene expression of $\mathrm{Ca}$. L. asiaticus in adult psyllids increased in adults feeding on CLasinfected plants for 1-2 d to 5-6 d. Thus, 725 transcripts of CLas were expressed in $\mathrm{AlCLas}^{+}, 766$ in $\mathrm{A2CLas}^{+}$ and 804 in $\mathrm{A3CLas}^{+}$(Fig. 1). One transcript was expressed only in $\mathrm{A1CLas}{ }^{+}$and $\mathrm{A2CLas}{ }^{+}, 22$ in $\mathrm{A1CLas}{ }^{+}$ and $\mathrm{A3CLas}{ }^{+}$, and 63 in $\mathrm{A2CLas}{ }^{+}$and $\mathrm{A3CLas}{ }^{+}$. We also identified CLas genes that were exclusively expressed at

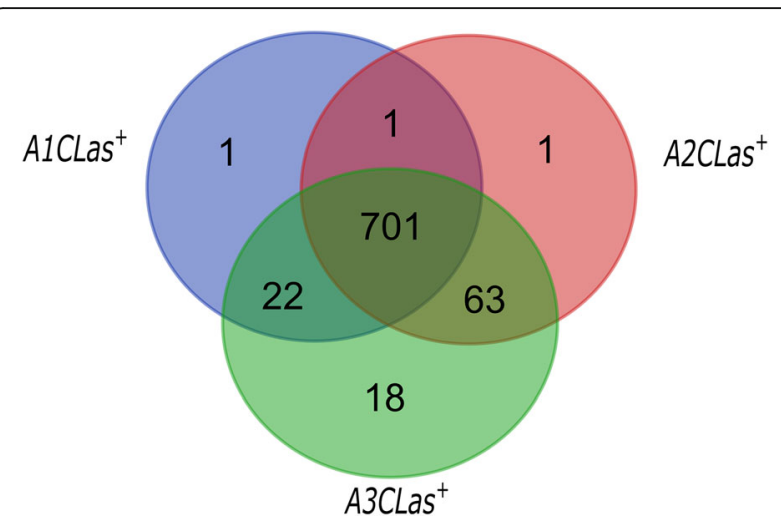

Fig. 1 Venn diagram of Candidatus Liberibacter asiaticus transcripts differentially expressed in gut of adults at different periods of feeding on CLas-infected citrus plant. A1CLas ${ }^{+}$: adults that fed on CLas-infected citrus plant for 1-2 days; $\mathrm{A2Clas}^{+}$: adults that fed on CLas-infected citrus plant for 3-4 days; and A3CLas ${ }^{+}$: adults that fed on CLas-infected citrus plant for 5-6 days.

the early $\left(1-2 \mathrm{~d}, \mathrm{AlCLas}^{+}=1\right.$ transcript, DN14421_c0 g2_i2 = nicotinate-nucleotide adenylyltransferase), intermediate (3-4 d, A2CLas ${ }^{+}=1$ transcript, DN18703_c1_ g1_i24 = Flp type IVb pilin) and late stage $(5-6 \mathrm{~d}$, $\mathrm{A3CLas}^{+}=18$ transcripts) after adult started feeding on CLas-infected citrus plants (Fig. 1). Most of these transcripts were represented by several isoforms of a gene, with different isoforms expressed in more than one of the sampled feeding times. Only $\mathrm{A}_{3 \mathrm{CLas}}{ }^{+}$adults had transcripts represented by unique isoforms of a gene specifically expressed in their gut (Table 1 ).

Pairwise gene expression analysis of CLas in the gut of adult psyllids after different periods of feeding on CLasinfected citrus plants revealed a high number of differentially expressed CLas genes in $\mathrm{A3CLas}^{+}$as compared to $\mathrm{AlCLas}^{+}$and $\mathrm{A2CLas}^{+}$adults (Additional file 3). Onehundred transcripts out of the over 700 transcripts detected in the three sampling times differed in their abundance (Additional file 3). Differences in the level of expression were detected for 80 transcripts when comparing $\mathrm{AlCLas}^{+}$and $\mathrm{A3CLas}^{+}$and 20 transcripts in comparisons of $\mathrm{A2CLas}^{+}$and $\mathrm{A3CLas}^{+}$(Additional file 3). CLas expression was always higher in $\mathrm{A}_{3} \mathrm{CLas}^{+}$when compared to the others. No differences in gene expression between $A 1 C \mathrm{Las}^{+}$and $\mathrm{A2C \textrm {Cas } ^ { + }}$ were detected (Additional file 3).

\section{Differential gene expression in the gut of $\mathrm{CLas}^{+}$and $\mathrm{CLas}^{-}$ adults of Diaphorina citri}

Differential gene expression (DE) of the gut of adults of D. citri after feeding on CLas-infected plants for 1-2 days identified 24 DE transcripts in the gut of $\mathrm{CLas}^{+}$insects, 20 of them were up-regulated and 4 were downregulated in response to CLas infection. Most of the upregulated transcripts (11) belong to uncharacterized 
Table 1 Single isoforms of CLas transcripts exclusively expressed in the gut of adult psyllids in A3CLas+

\begin{tabular}{lll}
\hline Transcript ID & Annotation & TPM count \\
\hline DN1443_C0_g1_i1 & hypothetical protein PSGCA5_05 & 58.77 \\
DN15414_C0_g1_i2 & pyridoxine 5'-phosphate synthase & 5.33 \\
DN25987_C0_g1_i1 & hypothetical protein & 30.77 \\
DN32802_C0_g1_i1 & anti-repressor protein & 38.31 \\
DN39169_C0_g1_i1 & hypothetical protein BWK56_05555 & 69.16 \\
DN44737_c0_g1_i1 & threonine--tRNA ligase & 168.28 \\
DN47430_C0_g1_i1 & flagellar basal body rod protein FlgF & 54.15 \\
DN55271_c0_g1_i1 & phage related protein & 10.66 \\
DN62541_c0_g1_i1 & flagellar basal body P-ring protein Flgl \\
DN64078_c0_g1_i1 & putative phage-related acetyltransferase & 31.40 \\
\hline
\end{tabular}

proteins, while nine up-regulated transcripts were identified (Table 2). After 3-4 days of feeding on CLasinfected plants, the number of DE transcripts in the gut of $\mathrm{CLas}^{+}$as compared to CLas adult psyllids increased to 35 , most of which were up-regulated (27). Eighteen out of the 27 up-regulated transcripts, and seven out of the eight down-regulated transcripts were putatively identified (Table 2). The number of DE transcripts in the gut of adult psyllids after 5-6 days of feeding on CLas-infected plants was the highest, with 61 DE transcripts in the gut of $\mathrm{CLas}^{+}$insects, 57 (33 unknown proteins) of them were up-regulated and 4 were downregulated when compared to CLas adult psyllids (Table 2).

From the total number of differentially expressed transcripts of $\mathrm{CLas}^{+}$when compared to their respective CLas ${ }^{-}$psyllids, only one transcript (DN16253_c6_g2_i4: uncharacterized protein LOC103515486) was differentially expressed in $\mathrm{CLas}^{+}$psyllids regardless the time they were allowed to feed on CLas-infected citrus plants as compared to psyllids fed on CLas-uninfected citrus plants (Fig. 2). Most of the DE transcripts detected in $\mathrm{CLas}^{+}$psyllids differed only at the specific stage they were compared. Seventeen (71\%) of the DE transcripts detected in the gut of adult psyllids after 1-2 d, 31 (88\%) after 3-4 d and 60 (88\%) after 5-6 d of feeding in CLasinfected plants differed from controls specifically at each particular stage (Fig. 2).

\section{Discussion}

The differential gene expression of the gut of adult psyllids differed depending on the duration of feeding on CLas-infected citrus plants. The number of DE transcripts in the gut of psyllids increased as the time the psyllids fed on CLas-infected plants increased. Most interesting, the majority of DE transcripts detected were specific to each one of the periods of feeding psyllids remained exposed to CLas-infected plants.
Gene expression of CLas in the gut of adult psyllids after different times of feeding on CLas-infected citrus plants were quite different. CLas transcription in the gut of adult psyllids was highly active soon after adult feeding started, but expression of a large set of genes was significantly increased at later stages of feeding $\left(\right.$ A3CLas $\left.^{+}\right)$.

\section{Immune attack and immune defense responses}

Bacteria that gain access to the hemocoel of insects find an easy way through the gut epithelium of the midgut, since the epithelium in this region does not have the cuticle lining protecting the fore- and the hindgut. Psyllids, as other sap-feeding insects do not carry the peritrophic membrane that protects the midgut epithelium of several other groups of insects [36, 37]. But regardless the presence of such physical barriers, the front line of defense of the gut immune system involves the activation of the epithelial innate immune system for the production of reactive oxygen species (ROS). Bacteria that survive in the gut of insects are either resistant to or are able to metabolize $\operatorname{ROS}[38,39]$.

Analysis of the CLas gene expression in the gut of adult psyllids demonstrated that CLas expressed the cytoprotective antioxidant enzyme peroxiredoxin (DN17546_c4_g1_i1), capable of reducing ROS and reactive nitrogen species (RNS) produced in the process of gut infection [40, 41]. Peroxiredoxins (EC 1.11.1.15) are cysteine-dependent peroxidases targeting hydrogen peroxide, peroxynitrite and organic peroxide as substrates [42]. Peroxiredoxins utilize either 2-Cys or 1-Cys reaction catalysis mechanisms [42], with CLas showing two chromosomal 1-Cys-1 peroxiredoxin genes (CLIBASIA RS00940 and CLIBASIA_RS00445). These genes are highly conserved in all CLas sequenced strains [42]. The $\mathrm{DE}$ expressed transcript detected in the gut of CLasinfected psyllids (DN17546_c4_g1_i1) shared 100\% identity with CLIBASIA_RS00940 (WP_012778530). But peroxidase activity has only been reported for CLIBASIA_ 
Table 2 Comparative differential gene expression of adult psyllids fed on CLas infected and non-infected citrus plants for different periods of time $(A 1=1-2 d ; A 2=3-4 d ; A 3=5-6 d)$

\begin{tabular}{|c|c|c|c|c|c|}
\hline Stage & $\mathrm{DE}$ & Annotation & Transcript ID & FC & FDR \\
\hline \multirow[t]{24}{*}{${ }^{*} \mathrm{~A} 1-\mathrm{CLas}^{+} \times \mathrm{Clas}^{-}$} & \multirow[t]{20}{*}{ Up-regulated } & arylsulfatase B-like isoform X1 & DN19237_c6_g3_i2 & 20.9 & $2.0 e^{-2}$ \\
\hline & & CLIP domain-containing serine protease 2 -like isoform X1 & DN16851_c1_g2_i2 & 119.9 & $3.0 e^{-3}$ \\
\hline & & kinesin-like protein KIF3B isoform X4 & DN10161_c0_g1_i1 & 55.2 & $6.1 e^{-3}$ \\
\hline & & membrane metallo-endopeptidase-like 1 isoform X2 & DN16798_c0_g1_i1 & 49.0 & $4.8 e^{-4}$ \\
\hline & & methylglutaconyl-CoA hydratase, mitochondrial & DN18628_c4_g1_i2 & 26.7 & $1.0 e^{-2}$ \\
\hline & & mitochondrial cytochrome c oxidase subunit IV & DN18774_c0_g1_i7 & 44.2 & $2.3 e^{-6}$ \\
\hline & & nuclear speckle splicing regulatory protein 1-like & DN17904_c2_g1_i3 & 133.9 & $4.0 e^{-2}$ \\
\hline & & uncharacterized protein LOC103515486 & DN16253_c6_g2_i4 & 11.3 & $9.64 e^{-3}$ \\
\hline & & protein phosphatase 1 regulatory subunit 21 & DN19112_c0_g1_i1 & 36.7 & 0.02 \\
\hline & & putative rna-directed dna polymerase from transposon bs & DN20114_C0_g1_i11 & 26.6 & 0.05 \\
\hline & & uncharacterized protein LOC103508016 & DN17144_c6_g5_i2 & 83.6 & $2.93 e^{-5}$ \\
\hline & & uncharacterized protein LOC103508016 & DN17144_c6_g5_i3 & 62.7 & 0.02 \\
\hline & & uncharacterized protein LOC103518490 & DN15191_c0_g1_i1 & 222.8 & $2.03 e^{-3}$ \\
\hline & & uncharacterized protein LOC103518490 & DN15191_C0_g1_i4 & 41.0 & $6.75 e^{-3}$ \\
\hline & & uncharacterized protein LOC103519237 & DN15191_c0_g1_i7 & 210.2 & $1.06 e^{-4}$ \\
\hline & & uncharacterized protein LOC113469268 & DN20635_c0_g3_i1 & 242.2 & $1.06 e^{-4}$ \\
\hline & & uncharacterized protein LOC113469268 & DN20635_c0_g1_i3 & 106.3 & $5.87 e^{-4}$ \\
\hline & & uncharacterized protein LOC113471433 & DN20585_c3_g1_i12 & 167.6 & $5.28 e^{-3}$ \\
\hline & & uncharacterized protein PFB0145c-like & DN15546_c0_g1_i1 & 31.3 & 0.04 \\
\hline & & uncharacterized protein YMR317W-like & DN15237_c0_g1_i1 & 132.6 & $4.69 e^{-4}$ \\
\hline & \multirow[t]{4}{*}{ Down-regulated } & NADH dehydrogenase [ubiquinone] 1 beta subcomplex subunit 3 & DN17549_c4_g1_i2 & -32.5 & $6.75 e^{-3}$ \\
\hline & & protein pangolin, isoforms $\mathrm{A} / \mathrm{H} / \mathrm{I} / \mathrm{S}$-like & DN15424_CO_g3_i2 & -28.0 & 0.03 \\
\hline & & titin isoform X7 & DN21409_c5_g2_i1 & -36.1 & 0.03 \\
\hline & & zonadhesin-like & DN14644_C0_g1_i1 & -13.0 & 0.03 \\
\hline \multirow[t]{19}{*}{${ }^{*} \mathrm{~A} 2-\mathrm{Clas}^{+} \times \mathrm{Clas}^{-}$} & \multirow[t]{19}{*}{ Up-regulated } & Ac1147-like protein & DN18394_c1_g1_i3 & 19.7 & 0.02 \\
\hline & & arylalkylamine $\mathrm{N}$-acetyltransferase & DN20634_C1_g1_i13 & 45.9 & 0.05 \\
\hline & & cathepsin L1-like & DN15698_c4_g11_i2 & 111.4 & $1.45 e^{-3}$ \\
\hline & & hypothetical protein DAPPUDRAFT_37179, partial & DN18534_c0_g5_i1 & 17.0 & $7.51 e^{-4}$ \\
\hline & & hypothetical protein g.47064 & DN18473_c2_g1_i1 & 11.7 & $8.68 e^{-3}$ \\
\hline & & hypothetical protein g.7198 & DN16128_c4_g1_i1 & 11.1 & $3.86 e^{-3}$ \\
\hline & & large neutral amino acids transporter small subunit 1 & DN20157_c2_g3_i2 & 66.6 & $1.50 e^{-4}$ \\
\hline & & nocturnin isoform $\mathrm{X} 1$ & DN15927_c10_g1_i6 & 15.1 & $2.82 e^{-4}$ \\
\hline & & predicted protein & DN21032_c17_g1_i2 & 27.0 & $2.19 e^{-3}$ \\
\hline & & predicted protein & DN21032_c17_g1_i1 & 23.9 & $6.41 e^{-3}$ \\
\hline & & uncharacterized protein LOC105736982 & DN21288_C9_g7_i2 & 28.5 & $8.64 e^{-4}$ \\
\hline & & uncharacterized protein LOC103515486 & DN16253_c6_g2_i4 & 12.6 & 0.04 \\
\hline & & profilin & DN19422_c2_g2_i1 & 162.4 & $7.00 e^{-9}$ \\
\hline & & protein hu-li tai shao & DN19744_c0_g2_i2 & 12.2 & 0.01 \\
\hline & & putative senescence-associated protein & DN18133_c2_g1_i3 & 28.0 & $1.41 e^{-3}$ \\
\hline & & Regulator of rDNA transcription protein 15 & DN16244_c2_g3_i1 & 24.8 & $1.62 e^{-3}$ \\
\hline & & Regulator of rDNA transcription protein 15 & DN21288_c9_g7_i1 & 16.4 & $6.43 e^{-3}$ \\
\hline & & Regulator of rDNA transcription protein 15 & DN16244_c2_g3_i2 & 15.6 & $7.58 e^{-4}$ \\
\hline & & Regulator of rDNA transcription protein 15 & DN16244_c2_g3_i3 & 13.0 & $5.46 e^{-3}$ \\
\hline
\end{tabular}


Table 2 Comparative differential gene expression of adult psyllids fed on CLas infected and non-infected citrus plants for different periods of time $(A 1=1-2 d ; A 2=3-4 d ; A 3=5-6 d)$ (Continued)

\begin{tabular}{|c|c|c|c|c|c|}
\hline Stage & $\mathrm{DE}$ & Annotation & Transcript ID & FC & FDR \\
\hline & & reverse transcriptase-like protein & DN21162_c2_g1_i4 & 12.4 & 0.02 \\
\hline & & sodium/potassium-transporting ATPase subunit alpha-like & DN14485_c0_g1_i1 & 17.9 & $5.33 e^{-4}$ \\
\hline & & UNC93-like protein MFSD11 & DN16539_c2_g6_i1 & 48.5 & 0.04 \\
\hline & & Uncharacterized protein ART2 & DN19721_c0_g1_i3 & 9.8 & 0.02 \\
\hline & & uncharacterized protein LOC105206152 & DN18133_c2_g1_i1 & 22.8 & $1.45 e^{-3}$ \\
\hline & & uncharacterized protein LOC105693632 & DN19721_C0_g4_i1 & 7.9 & $8.56 e^{-3}$ \\
\hline & & uncharacterized protein LOC113219380 & DN19326_c1_g1_i2 & 35.7 & $6.21 e^{-4}$ \\
\hline & & uncharacterized protein LOC113469268 & DN20635_c0_g1_i1 & 37.4 & 0.02 \\
\hline & Down-regulated & leptin receptor overlapping transcript-like 1 & DN20058_c2_g3_i4 & -26.7 & 0.02 \\
\hline & & methylglutaconyl-CoA hydratase, mitochondrial & DN18628_c4_g1_i2 & -11.3 & $1.27 e^{-3}$ \\
\hline & & putative juvenile hormone binding protein & DN21423_c1_g12_i2 & -12.3 & 0.04 \\
\hline & & Soluble NSF attachment protein & DN21196_c2_g1_i1 & -19.8 & $5.64 e^{-7}$ \\
\hline & & translation elongation factor 2 & DN21188_c1_g7_i2 & -11.9 & 0.02 \\
\hline & & tubulin-folding cofactor B & DN17504_c3_g2_i6 & -4.5 & $1.82 e^{-3}$ \\
\hline & & uncharacterized protein LOC103513992 & DN16335_c5_g1_i1 & -16.7 & $5.18 e^{-3}$ \\
\hline & & zinc transporter ZIP10 isoform X1 & DN19132_c8_g1_i5 & -43.4 & 0.02 \\
\hline \multirow[t]{27}{*}{ ***A3 $-\mathrm{CLas}^{+} \times \mathrm{Clas}^{-}$} & Up-regulated & 2-oxoglutarate dehydrogenase, mitochondrial-like & DN14833_c0_g1_i1 & 8.6 & 0.03 \\
\hline & & Ac1147-like protein & DN21090_c8_g2_i1 & 50.3 & 0.04 \\
\hline & & BCL2/adenovirus E1B 19 kDa protein-interacting protein 3 & DN20168_c2_g3_i4 & 21.0 & 0.04 \\
\hline & & beta-1,3-galactosyltransferase 5 -like & DN18243_c2_g2_i2 & 20.2 & $7.31 e^{-3}$ \\
\hline & & cleavage and polyadenylation specificity factor subunit CG7185-like & DN15201_C0_g1_i1 & 15.6 & $6.05 e^{-3}$ \\
\hline & & cytochrome P450-like TBP & DN19721_C0_g1_i4 & 35.7 & 0.05 \\
\hline & & dynein heavy chain 7 , axonemal-like & DN35915_c0_g1_i1 & 17.3 & 0.05 \\
\hline & & gamma-glutamyl hydrolase-like & DN18300_c5_g1_i2 & 16.7 & $3.96 e^{-5}$ \\
\hline & & GATA zinc finger domain-containing protein 14-like & DN16387_c3_g2_i1 & 12.2 & $6.66 e^{-3}$ \\
\hline & & glycerophosphocholine phosphodiesterase GPCPD1-like isoform X1 & DN15741_CO_g2_i2 & 41.6 & $1.10 e^{-4}$ \\
\hline & & hydrocephalus-inducing protein homolog & DN14616_c0_g1_i2 & 26.6 & 0.03 \\
\hline & & hydrocephalus-inducing protein homolog & DN14616_c0_g2_i2 & 16.6 & 0.02 \\
\hline & & hypothetical protein ALC57_18598 & DN17391_c0_g1_i2 & 230.6 & $1.56 e^{-4}$ \\
\hline & & hypothetical protein FF38_03795 & DN18516_c0_g3_i1 & 67.0 & 0.02 \\
\hline & & hypothetical protein LOTGIDRAFT_202939 & DN17968_c0_g2_i3 & 101.2 & 0.03 \\
\hline & & hypothetical protein Phum_PHUM590900 & DN17430_c2_g2_i1 & 24.2 & 0.02 \\
\hline & & keratin-associated protein 5-3-like & DN15230_C0_g1_i2 & 14.2 & $6.76 e^{-4}$ \\
\hline & & keratinocyte proline-rich protein-like & DN20284_C2_g1_i3 & 11.5 & 0.02 \\
\hline & & mucin-5AC-like, partial & DN16207_c6_g5_i1 & 7.7 & 0.04 \\
\hline & & uncharacterized protein LOC103511862 & DN17183_c5_g2_i3 & 9.4 & 0.04 \\
\hline & & uncharacterized protein LOC103513455 & DN17270_c3_g2_i1 & 46.8 & 0.04 \\
\hline & & uncharacterized protein LOC103514633 & DN18467_c1_g5_i1 & 31.7 & $3.61 e^{-5}$ \\
\hline & & uncharacterized protein LOC103515486 & DN16253_c6_g2_i4 & 12.6 & 0.04 \\
\hline & & uncharacterized protein LOC103518002 & DN18156_c6_g2_i2 & 10.0 & 0.03 \\
\hline & & uncharacterized protein LOC103521603 & DN17270_c3_g2_i2 & 63.7 & 0.01 \\
\hline & & uncharacterized protein LOC103521674 & DN15182_c0_g1_i1 & 9.3 & 0.03 \\
\hline & & protein AMN1 homolog & DN15340_c0_g1_i1 & 8.9 & 0.04 \\
\hline
\end{tabular}


Table 2 Comparative differential gene expression of adult psyllids fed on CLas infected and non-infected citrus plants for different periods of time $(A 1=1-2 d ; A 2=3-4 d ; A 3=5-6 d)$ (Continued)

\begin{tabular}{|c|c|c|c|c|c|}
\hline Stage & DE & Annotation & Transcript ID & FC & FDR \\
\hline & & protein FAM133-like isoform X1 & DN19474_c1_g3_i11 & 15.4 & $8.40 e^{-3}$ \\
\hline & & protein PRRC2C-like & DN15390_c0_g1_i1 & 21.0 & $3.47 e^{-4}$ \\
\hline & & protein suppressor of forked & DN20501_c1_g1_i2 & 50.7 & 0.04 \\
\hline & & putative juvenile hormone binding protein & DN21423_c1_g12_i1 & 33.0 & $5.4 e^{-5}$ \\
\hline & & putative juvenile hormone binding protein & DN21423_c1_g12_i2 & 9.4 & $8.34 e^{-3}$ \\
\hline & & RNA-directed DNA polymerase from mobile element jockey-like & DN19725_c3_g1_i3 & 14.1 & $6.95 e^{-3}$ \\
\hline & & senescence-associated protein & DN16041_c1_g1_i2 & 198.7 & $1.67 e^{-4}$ \\
\hline & & SWI/SNF chromatin-remodeling complex subunit SNF5-like & DN18520_c6_g1_i1 & 52.5 & $1.18 e^{-5}$ \\
\hline & & uncharacterized protein Dyak_GE27401 & DN16348_c2_g1_i1 & 18.0 & 0.02 \\
\hline & & uncharacterized protein LOC103507494 isoform X2 & DN20548_c5_g2_i7 & 13.1 & 0.01 \\
\hline & & uncharacterized protein LOC103507496 & DN20260_c2_g2_i2 & 17.0 & $6.6 e^{-4}$ \\
\hline & & uncharacterized protein LOC103507787 & DN20585_c3_g1_i2 & 60.0 & $8.58 e^{-3}$ \\
\hline & & uncharacterized protein LOC103512709 & DN20458_c5_g2_i2 & 29.7 & 0.03 \\
\hline & & uncharacterized protein LOC103514651 & DN18692_c1_g1_i7 & 41.1 & $9.13 e^{-4}$ \\
\hline & & uncharacterized protein LOC103516995 & DN14754_C0_g3_i1 & 39.3 & $2.89 e^{-3}$ \\
\hline & & uncharacterized protein LOC103518490 & DN15191_c0_g1_i2 & 105.3 & 0.05 \\
\hline & & uncharacterized protein LOC103518490 & DN15191_C0_g1_i4 & 85.7 & $7.47 e^{-3}$ \\
\hline & & uncharacterized protein LOC103519237 & DN15191_c0_g1_i7 & 36.9 & 0.01 \\
\hline & & uncharacterized protein LOC103520317 & DN17514_c8_g2_i1 & 10.7 & 0.01 \\
\hline & & uncharacterized protein LOC103524424 & DN14888_c0_g2_i1 & 7.8 & 0.04 \\
\hline & & uncharacterized protein LOC108252545 isoform X1 & DN16387_c3_g1_i9 & 7.6 & 0.03 \\
\hline & & uncharacterized protein LOC113219351 & DN17193_c2_g1_i6 & 74.5 & $8.74 e^{-4}$ \\
\hline & & uncharacterized protein LOC113219380 & DN18715_c0_g1_i6 & 305.6 & $9.53 e^{-5}$ \\
\hline & & uncharacterized protein LOC113219380 & DN21090_C7_g10_i1 & 82.0 & 0.04 \\
\hline & & uncharacterized protein LOC113469268 & DN20635_c0_g3_i2 & 73.8 & 0.03 \\
\hline & & uncharacterized protein LOC113469268 & DN20635_c0_g1_i1 & 37.4 & 0.02 \\
\hline & & uncharacterized protein LOC113469268 & DN20635_c0_g3_i1 & 36.9 & 0.02 \\
\hline & & uncharacterized protein LOC113469388 & DN14497_c0_g1_i2 & 8.9 & 0.04 \\
\hline & & uncharacterized protein YMR317W-like & DN15237_c0_g1_i1 & 60.2 & $8.52 e^{-4}$ \\
\hline & & Zgc:165536 protein & DN19721_c0_g2_i1 & 186.9 & $8.52 e^{-4}$ \\
\hline & \multirow[t]{4}{*}{ Down-regulated } & annexin B9-like isoform X1 & DN19784_C8_g1_i2 & -11.0 & 0.03 \\
\hline & & cytosolic purine 5'-nucleotidase isoform X4 & DN16811_c2_g1_i5 & -37.4 & 0.02 \\
\hline & & probable 4-coumarate--CoA ligase 1 & DN19422_C2_g3_i12 & -7.4 & 0.05 \\
\hline & & UDP-glucuronosyltransferase 2C1-like & DN20095_c3_g1_i5 & -12.4 & $1.92 e^{-3}$ \\
\hline
\end{tabular}

* A1-CLas+ $x$ CLas-: Differential gene expression of adults with 1 to 2 days of feeding; ** A2-CLas+ $x$ CLas-: Differential gene expression of adults with 3 to 4 days of feeding; ${ }^{* * *}$ A3- CLas+ $x$ CLas-: Differential gene expression of adults with 5 to 6 days of feeding

RS00445 (WP_012778432.1) [42, 43]. The CLas BCP endoded by this gene was demonstrated to be an extracellular 1-Cys peroxiredoxin that suppresses the signaling and accumulation of oxypilins in plants mediated by the respiratory burst oxidase homologs (RBOH). Regulation of this process was reported as essential for CLas survival and host plant colonization [42, 43].

The significant increase in the expression of peroxiredoxin from $\mathrm{AlCLas}{ }^{+}$to $\mathrm{A3CLas}{ }^{+}(\mathrm{FC}=13.48)$ suggests this enzyme provides an increased contribution in the infection process, as CLas cells also established an intracellular interaction with the gut epithelium of psyllids. CLas expression of peroxiredoxin occurred despite the expected reduction of ROS in the gut epithelium of adult psyllids, as the major source for ROS production in the gut, NADH dehydrogenase [ubiquinone] 1 beta subcomplex subunit 3 (DN17549_c4_g1_i2), was downregulated in $\mathrm{CLas}^{+}$psyllids. 


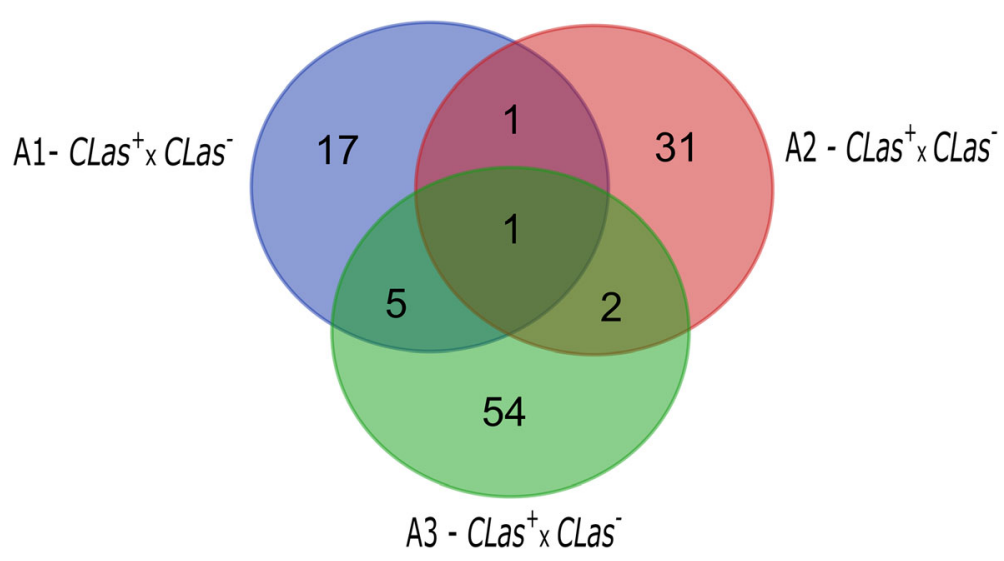

Fig. 2 Venn diagram of Diaphorina citri transcripts differentially expressed in gut of adults at different periods of feeding on CLas-infected citrus plant compared to insects that fed on healthy citrus plant. A1 - CLas ${ }^{+} \times \mathrm{Clas}^{-}$: adults that fed on CLas-infected versus uninfected citrus plant for 1-2 days; A2 - CLas ${ }^{+} \times$CLas: adults that fed on CLas-infected versus uninfected for 3-4 days; and A3 - CLas ${ }^{+} \times$CLas: $^{-}$adults that fed on CLasinfected versus uninfected for 5-6 days.

These results suggest that CLas can inhibit hydrogen peroxide production in the host insect, and that peroxiredoxin is certainly serving roles other than its antioxidative contribution. The adipokinetic hormone (ADK) is involved in the regulation of the oxidative stress response in insects [44]. Unfortunately, the expression levels of ADK-related genes by CLas infection could not be verified as ADK is produced and stored in neurosecretory cells of the corpora cardiaca. Nevertheless, regulation of this neuropeptide hormone in the gut epithelium of $\mathrm{CLas}^{+}$psyllids could occur by the upregulation of the metallo-endopeptidase-like 1 isoform X2 (DN16798_c0_g1_i1) neprilysin. Neprilysins are metalloproteases better known for their neuropeptide degrading activity, while also carrying peptide-degrading activity in other tissues, including the gut [45].

CLas peroxiredoxin could also be acting in the regulation of $\mathrm{H}_{2} \mathrm{O}_{2}$-mediated cell signaling processes, such as those involved in growth and immune responses [46]. The oxidative response produced by gut epithelia due to microbial infection leads to cell proliferation and modulation of the innate immune response [47], and ROS is required for inducing nitric oxide production in the gut. Nitric oxide production will in turn trigger the synthesis of antimicrobial peptides (AMP) and activate organ-toorgan communication [48]. Therefore, the downregulation of ROS production in the gut of $\mathrm{CLas}^{+}$psyllids could explain the lack of differential expression of genes belonging to the AMP production pathways. AMP synthesis can also be elicited by the recognition of peptidoglycans released by bacteria by membrane-bound peptidoglycan recognition proteins if peptidoglycans survive to amidase degradation [39].

There is very little information on insect gut phagocytosis, although proteomic analysis of anal droplets of the beetle Cryptorhynchus lapathi larvae led to the identification of several proteins to support an immune cellular response at the gut level [49]. Moreover, phagocytic receptors identified in the gut epithelium of Drosophila were proven to play crucial role in the phagocytosis of both Gram bacteria, and in controlling hemocele infection by bacteria invasion through the gut epithelium [50]. We could not detect any changes at the molecular level in $\mathrm{CLas}^{+}$psyllids that would demonstrate a phagocytic response in the gut, as well as we did not observe adult psyllids to build an immune response to CLas infection by activating ROS and AMP pathways.

Nevertheless, differential gene expression analysis of the gut of $\mathrm{AlCLas}{ }^{+}$psyllids demonstrated the mounting of a defensive response of the gut epithelium based on the increased expression of a CLIP domain-containing serine protease 2-like (DN16851_c1_g2_i2). CLIP domain-containing serine protease 2-like has been recently reported to be up-regulated in the midgut of CLas-infected psyllids [29]. Serine proteases containing a CLIP domain are involved in the regulation of humoral responses through the activation of prophenoloxidases (PPO) and the Toll immune signaling pathway. Activation of Toll pathway leads to antimicrobial peptides production, while the activation of PPO results in melanogenesis [51, 52]. Toll activation requires a multistep proteolytic cascade [53], and the lack of additional differentially expressed serine proteases and the antimicrobial peptides transcripts in the psyllid transcriptome support the argument that the up-regulation of CLIP-domain serine protease in the gut of ACP feeding on CLas-infected plants leads to the activation of melanization as an immune response against CLas infection. We also believe the activation of melanization at this stage of psyllid-CLas interaction was triggered by a local 
response caused by cell injury and invasion of the gut epithelium by CLas cells, resulting in the production of wound clots in order to avoid infected, damaged cells to suffer further damage, die and be replaced by nidi cells. This hypothesis is also supported by the downregulation of pangolin (Pan) (DN15424_c0_g3_i2), a key regulator of the $\mathrm{Wnt} / \mathrm{Wg}$ pathway. Wnt proteins are highly conserved and participate in the control of growth, patterning, tissue and energy homeostasis. Pangolin was reported to bind to the $\beta$-catenin homologue Armadillo, acting directly in the regulation of gene expression in response to Wnt signaling $[54,55]$. The Wnt signaling in Drosophila is an important process for the homeostasis of the gut tissue as it is involved in the regeneration of adult gut epithelial cells $[56,57]$. Thus, the down-regulation of pangolin interferes with the activation of the Wnt signaling to regulate gene expression involved in the replacement of CLas-infected cells by the activation of the nidi cells to differentiate into new, active cells of the gut epithelium.

Studies on the role of genotype-genotype interactions of insects and parasites demonstrated activation and differential gene expression of the host immune machinery depending on the interacting parasite genotype. Alternative splicing was also reported as a required element in the specificity of the immune response of insects to specific parasite genotypes [58]. The nuclear speckles carry pre-mRNA splicing machinery composed by nuclear speckle-related proteins that mediate alternative splice site selection in targeted pre-mRNAs $[59,60]$. The detected up-regulation of proteins that mediate alternative splice site selection in targeted mRNAs, the nuclear speckle splicing regulatory protein 1-like (DN17904_c2_ g1_i3) in $\mathrm{CLas}^{+}$psyllids, suggests CLas acts on the regulation of gene expression of infected psyllids at the molecular level.

The psyllid initial defense response against CLas infection also included the up-regulation of arylsulfatase B (DN19237_c6_g3_i2), an enzyme stored in lysosomes that acts on large glycosaminoglycan molecules by removing attached sulfate groups [61]. Glycosaminoglycans are components of proteoglycans commonly exploited by pathogens as receptors for their adherence to different tissues [62]. Glycosaminoglycans are produced by some pathogenic bacteria as extracellular capsule and used in the process of host infection and colonization to facilitate pathogen attachment, invasion and/or evasion of host defensive mechanisms [63]. Arylsulfatases B can also participate in processes of biotransformation, mediating the sulfonation of xenobiotics by other enzymes to facilitate their excretion [64]. In this case, increase in the arylsulfatase could be related to the psyllid physiological needs to metabolize the high levels of flavonoids (hesperidin, narirutin and dydimin) produced in citrus plants infected by $\mathrm{Ca}$. Liberibacter asiaticus [65-67]. Flavanoids are important metabolites produced by plants in response to abiotic and biotic stressors and play a relevant contribution in plant resistance against microbes and herbivores [68], including citrus plants in response to CLas infection $[69,70]$.

The overexpression of immune related genes and the required increased cell metabolism early in the process of interaction of the psyllid gut epithelium with CLas can explain the up-regulation of the mitochondrial cytochrome c oxidase subunit IV (DN18774_c0_g1_i7) in $\mathrm{CLas}^{+}$insects. Mitochondrial cytochrome c oxidase subunit IV is the major regulation site for oxidative phosphorylation by catalyzing the final step of electron transfer in mitochondria [71].

\section{Cross-talk in the colonization of the gut lumen}

Bacteria that survive the harsh chemical environment and the immune barriers available in the gut will have to use strategies to avoid their rapid elimination from the gut with faeces [39, 72]. Insects are active feeders and food fastly transits through the gut. The fast transit of the food in the gut has been argued as one condition to explain the controversial lack of a resident microbiome in lepidopteran larvae, for example [73].

CLas seems to employ different strategies to colonize the gut lumen by avoiding its elimination with faeces: regulation of gut peristalsis, synthesis of adherence proteins, and biofilm formation.

CLas regulation of the psyllid gut peristalsis is thought to occur through the up-regulation of the host neprilysin as earlier discussed. Neprilysins are reported to degrade peptide hormones, and the gut of insects contains several hormone-producing cells. Peptide hormones produced in the gut act as signaling molecules that are involved in the regulation of a range of processes, including gut peristalsis [74-76]. The hypothesis that CLas modulates the gut peristalsis of psyllids is supported by the down-regulation of titin (DN21409_c5_g2_i1) transcription in $\mathrm{CLas}^{+}$psyllids. Muscle degeneration is marked by a reduction in titin proteins, and gutassociated muscles are in charge of producing the peristaltic movement observed in the gut. Additionaly, the intracellular non-catalytic domain of neprilysins in excess is shown to induce muscle degeneration [77]. Thus, the degeneration of muscles associated with the gut would certainly impair gut peristalsis and consequently reduce the elimination of bacteria with faeces [39, 72].

Regulation of signaling in the gut is also evidenced by the up-regulation of arylalkylamine $\mathrm{N}$-acetyltransferase (DN20634_c1_g1_i13) transcription in $\mathrm{A2CLas}^{+}$psyllids. Arylalkylamine $\mathrm{N}$-acetyltransferases are involved in the $\mathrm{N}$-acetylation of arylalkylamines, playing an important role in the synthesis of melatonin in vertebrates and 
invertebrates [78, 79]. But in insects, arylalkylamine Nacetyltransferases also inactivate arylalkymines that play important roles as neuromodulators, such as octopamine, dopamine and serotonin [80, 81]. These neuromodulators are also implicated in the double brain-gut communication circuitry $[82,83]$.

CLas adherence to the psyllid gut was achieved by the expression of three genes of a subtype of the type IVb pilins, the tight adherence pili $(\mathrm{Tad}=\mathrm{Flp})$. Flp pilins are common to several $\mathrm{Gram}^{+}$and $\mathrm{Gram}^{-}$bacteria. Type IV pili are highly diverse and involved in a number of protein-protein interactions, but flp pili are better known for their role in adherence to living and nonliving surfaces. Pili are also involved in cell motility, secretion of exoproteins, and in host cell manipulation under extreme conditions [84, 85].

The expression of Type IVc pili of Ca. L. asiaticus was reported to be higher in psyllids than in plants, but only one (flp3 pilin) out of five flp genes was reported to be up-regulated in psyllids [86]. The flp3 pilin gene was demonstrated to be under the regulation of VisN and VisR, demonstrating these proteins are important in the colonization of the host vector [86]. In our analysis of the transcriptional profile of CLas in the gut of adult psyllids, we detected the expression of several isoforms of two flp genes (DN14425_c0_g1; DN18703_c0_g1), but both belonging to the family Type IVb pilin. Three isoforms of gene DN14425_c0_g1 (DN14425_c0_g1_i2; DN14425_c0_g1_i3; DN14425_c0_g1_i4) and two of gene DN18703_c0_g1 (DN18703_c0_g1_i21 and DN18703_c0_g1_i23) were highly and consistently expressed in the gut of psyllids in all sampling periods. Three CLas LuxR family transcriptional regulators (DN1177_c0_g1_i1, DN14940_c0_g2_i1, and DN38633 c0_g1_i1) were also observed in the gut of $\mathrm{CLas}^{+}$psyllids; one of them refers to the VisR regulator (DN1177_ c0_g1_i1 = CLIBASIA_02900), and the other two to the VisN regulator (DN14940_c0_g2_i1 and DN38633_c0_ g1_i1 = CLIBASIA_02905) of flp3 pilin gene reported by Andrade \& Wang [86].

The expression of CLas flagellins (FlgA) (DN3624_c0_ g2_i1 and DN40834_c0_g1_i1) and several genes involved in the assembling and functioning of the flagellar machinery (FliE, FliF, FliG, FliK, FliN, FliR, FliP, FlgB, FlgD, FlgE, FlgF, FlgG, FlgH, FlgI, FlgK, FlhA, FlhB, $M o t B$, and the flagellar $C$-ring protein) would indicate CLas assembles flagella for colonizing the gut of adult psyllids, although FlgF and FlgI were detected exclusively in $\mathrm{A3CLas}^{+}$. The expression of genes involved in the flagellar machinery corroborates recent data on the higher expression of genes encoding the flagellum apparatus of CLas in the gut of psyllids than in plants [87]. Although we detected a much higher number of transcripts of the flagellar system of the CLas isolate we worked with than those reported to be expressed in the gut of psyllids by Andrade et al. [87], we did not detect the expression of three genes they evaluated (fliQ, fliL, and $f l g A)$. Differences in the overall number of genes of the flagellar system among lineages are expected to occur, but not among isolates of the same species [88].

The production of flagellum in the gut by infecting CLas is also supported by transmission electron microscopy images, although most of the CLas cells observed lacked a flagellum [87]. Analysis of images of the flagellum of CLas cells clearly shows CLas carries the secondary flagellar system, producing a lateral flagellum. The secondary flagellar systems arose twice in the evolution of bacteria, once in alpha-proteobacteria and once in the common ancestor of beta/gamma-proteobacteria [89]. The primary flagellar system produces a polar flagellum and contributes with cell motility in liquid media, while the secondary flagellum system produces a lateral flagellum that is involved in adhesion and cell swarming on surfaces. Bacterial flagella serve bacteria not only as a motor apparatus, but also as a protein export/assembly apparatus. The motor apparatus of flagella can provide bacteria the movement required to remain in the gut, as demonstrated in the trypanosomatid Vickermania [90]. Moreover, the flagellum pattern can be altered by bacteria depending on the environmental conditions faced, and bacterial flagella are recognized as important virulence factors [91-93]. Chemotaxis is an important contribution of bacterial flagellum to virulence [94], and the detection of the expression of four chemotaxis protein genes (DN15199, DN31800, DN46197 and DN50630) suggests the flagellum participates in cell adhesion to and swarming on the psyllid gut epithelium. Yet, we also propose the flagellum aids CLas to become chemically oriented for the localization of suitable host cells for invasion.

Biofilm formation requires bacteria to synthesize an extracellular matrix. Biofilms include extracellular proteins, cell surface adhesins and subunits of flagella and pili proteins [95]. We did not identify the expression of adhesins in the transcriptome of CLas in the gut of adult psyllids. Nevertheless, several chaperones were highly expressed, and many chaperones can act as adhesins in bacteria. Additionally, we believe the cold shock protein (DN10086_c0_g2_i1) of CLas is also playing key roles in biofilm formation. Cold shock proteins were demonstrated not only to participate in biofilm formation but also to support cell adhesion and motility, and to stimulate cell aggregation, interfering thus with virulence $[96,97]$.

\section{Cross-talk for infecting the gut epithelium}

Several gut-associated bacterial symbionts of hemipterans enter in close contact with epithelial cells to establish an intracellular phase through endocytosis [98]. 
CLas also enters the gut epithelium of psyllids through endocytosis, remaining inside vacuoles formed from or surrounded by endoplasmic reticulum membrane [99]. The differential gene expression analysis of the gut of $\mathrm{CLas}^{+}$psyllids led to the identification of transcripts involved with ultrastructural alterations of the gut epithelial cells, allowing the identification of the molecular intermediates that participate in the route CLas follows to invade the gut epithelium.

In addition to the regulation of the immune related responses earlier described in the gut epithelium of $\mathrm{CLas}^{+}$ psyllids, epithelial cells of the gut were altered early in the infection process (A1CLas ${ }^{+}$adults). Alterations are perceived by the transcriptional down-regulation of zonadhesin-like (DN14644_c0_g1_i1), a transcriptional response already reported by Ramsey et al. [100]. Zonadhesin-like proteins represent an expansion of the zonadhesin multi-domain proteins that are implicated in the binding of sperm and egg in a species-specific manner. In the gut, zonadhesin-like proteins are thought to function as mucins, and two zonadhesin-like proteins of D. citri were previously predicted to be part of the extracellular matrix and to have a role in cell-cell adhesion. Mucins protect the gut epithelium from microbial infections and inflammation [101]. Mucins are components of the perimicrovillar layer that in psyllids and other paraneopterans [102] replaces the chitin-based matrix (peritrophic membrane) that provides a protective barrier to the gut epithelial cells from pathogenic bacteria [103].

In vertebrates the mucin composition of the mucous layer can vary among gut regions, with diet composition and gut microbial infection [104]. Mucins can also be exploited as a nutritional resource by bacteria, and degradation of mucins by a commensal microbe was demonstrated to facilitate the penetration of the epithelium by viruses $[105,106]$. The role different mucins can have on the host-pathogen interaction can explain the upregulation of the psyllid gene coding for mucin-5AC (DN16207_c6_g5_i1) in A3CLas ${ }^{+}$psyllids. The higher abundance of this protein in $\mathrm{CLas}^{+}$psyllids has been demonstrated in previous proteomic analysis [107]. Regulation of the host mucin-5AC has been reported in vertebrate hosts infected by $\mathrm{Gram}^{-}$and $\mathrm{Gram}^{+}$bacteria, and in one system modulation of mucin-5AC has been linked with increased adhesion of bacteria to the gut tissue $[108,109]$.

The differential expression of genes coding for cytoskeletal proteins involved in the regulation of the submembranous actin-spectrin network in cells of the gut epithelium of $\mathrm{CLas}^{+}$psyllids demonstrates CLas interferes with the remodeling of gut epithelial junctions in adult psyllids, leading to endocytosis of intercellular junctions with cellular organelles. Adducins (protein
Hu-li tai shao - DN19744_c0_g2_i2) form connections with membranes, promote spectrin-actin interactions and regulate actin filaments [110]. Internalization of apical junction and/or tight junction proteins may disrupt the epithelial barriers and favor the movement of bacterial toxins into cells [111].

The intracellular events in preparation for endocytosis can also be detected as indicated by the up-regulation of the psyllid phosphatase 1 regulatory subunit 21 (DN19112_c0_g1_i1) in the gut of A1CLas ${ }^{+}$. This protein participates in early (sorting process) or late (maturation) endosome pathway, which leads to the endocytosis of several types of materials, including pathogenic agents. Our hypothesis is that early endosomes will also internalize the leptin receptor overlapping protein-like 1 . The leptin receptor overlapping transcript-like 1 (DN20058_c2_g3_i4) was downregulated in the gut epithelium of $\mathrm{A2CLas}^{+}$psyllids. Internalization of such receptors in early endosomes also affects cell signaling, and in this particular case can severely interferes with the gut epithelium resistance to microbial infection. Down-regulation of leptin/leptin receptor was reported to drastically affect cell resistance to amoeba and bacterial infection in several species [112$114]$.

The endocytic vesicles detach and become free endocytic carrier vesicles transporting their cargoes to late endosomes to finally fuse with endoplasmic reticulum, where CLas cells aggregate in associated vacuoles as indicated by ultrastructural analysis [99]. Fusion of lysosomes to cell aggregates vacuoles is inhibited by the down-regulation of soluble NSF attachment protein (SNAPs) (DN21196_c2_g1_i1) in A2CLas ${ }^{+}$. SNAPs are highly conserved proteins that participate in intracellular membrane fusion and vesicular trafficking [115]. Intracellular membrane fusion requires both SNAPs and NSF to act in concert, and inhibition of one of them will lead to the failure of membrane fusion and the accumulation of vesicles one cannot fuse [116].

SNAPs are characterized by the presence of a tetratricopeptide repeat (TPR) domain [117]. Tetratricopeptide repeat proteins (TRP) are directly involved in virulence, particularly due the translocation of virulence factors into host cells and in the blockage of phagolysosomal maturation, among others [118]. Three TRPs (DN11192_c0_g1_i1; DN11192_c0_g1_i2; DN14826_c0_ g1_i2) were expressed in the gut of $\mathrm{CLas}^{+}$psyllids, but only DN14826_c0_g1_i2 was differentially expressed. The expression of different TRPs in all sampling periods and their time-specific differential expression indicates TRPs participates in different processes of CLas interactions with the host cells, from CLas establishment in the gut lumen to epithelial cell invasion and access to the hemocoel. 
The down-regulation of the psyllids annexin B9-like isoform X1 (DN19784_c8_g1_i2) in the gut of A3CLas ${ }^{+}$ would interferes with the development of multivesicular bodies, once annexin B9 is involved in endosomal trafficking to multivesicular bodies [119]. This change will affect the transfer of the contents of CLas-containing endosomal vesicles to lysosomes for proteolytic degradation.

We propose that the establishment of the intracellular cycle and survival of CLas within host cells was putatively aided by the expression of CLas peptidyl-prolyl isomerase (PPI) (DN14386_c0_g1_i1), a protein that has been proved to participate in intracellular infection and virulence of other Gram ${ }^{-}$bacteria [120-122]. The Mpi PPI from Legionella pneumophila was demonstrated to require an active enzymatic site to enhance a proper host cell invasion [123], although the preservation of the active site was not a requirement for the PPI of Burkholderia pseudomallei [120]. Ghanim et al. [99] suggested earlier that CLas cells do not enter the ER, but instead CLas cells recruit ER to transform the phagosome into a host-immune free space suitable for CLas survival and multiplication. These same ER-derived structures were already observed in Legionella and Brucella [124, 125].

Early endosomes and endoplasmic reticulum share contact sites that are used to bind to microtubules at or close to their contact sites, and both organelles remain bounded as endosomes traffic and mature [126]. Endosome trafficking within the cell involves the membrane binding to motor proteins and its transport along the actin and microtubule cytoskeleton [127]. Trafficking of endosomes in $\mathrm{CLas}^{+}$psyllids is provided by the upregulation of kinesin-like protein KIF3B isoform X4 (DN10161_c0_g1_i1) in the gut of psyllids. Kinesins are motor proteins involved in the movement of multiple cytoplasmic organelles [127].

Profilin (DN19422_c2_g2_i1), another protein involved in intracellular movement of organelles, was also up-regulated in the gut of $\mathrm{A2CLas}^{+}$psyllids. Profilins are actin-binding proteins capable of regulating actin polymerization and the availability of the actin cytoskeleton for binding to the endosomes; but new roles for profilins are being identified in vertebrates [128]. Additionally, profilin 1 plays an important role in host-pathogen interactions. Intracellular pathogens such as Listeria monocytogenes and Shigella flexneri use the host-cell actin cytoskeleton to propel themselves through the cytoplasm and to spread to neighboring cells without entering the extracellular space [128, 129]. Dynein (DN35915_c0_ g1_i1), another motor protein, was up-regulated in the gut of $\mathrm{A}_{3 \mathrm{CLas}^{+}}$psyllids. Dyneins also contribute to microtubulebased transport in eukaryotic cells [130-132].

\section{Cross-talk for moving to the hemocoel}

After CLas inhibits the early immune response and invades the intracellular space of the gut epithelium by regulating a clathrin-independent endocytosis mechanism, hiding from the host's immune activators within vacuoles surrounded by endoplasmic reticulum membrane, where CLas multiplies, the molecular mechanism behind the release of CLas bacteria from epithelial cells in the hemocoel may require additional data.

The higher expression in $\mathrm{ACCLas}^{+}$of seven (DN15630_c0_g2_i3 = CLIBASIA_00255; DN15330_c0_ g1_i2 = CLIBASIA_00880; DN14809_c0_g1_i = CLIBASIA_03170; DN14146_c0_g1_i1 = CLIBASIA = 04410; DN10141_c0_g1_i1 = CLIBASIA_00995; DN15141_c0_ g1_i1 = CLIBASIA_01620; DN15414_c0_g1_i4 = CLIBASIA_01605) out of the 67 Sec-dependent proteins common to Candidatus Liberibacter species [133] indicates these candidate effector proteins play an important role in the dynamics of CLas infection of the psyllid gut epithelium at this stage. In fact, genomic analysis of Liberibacter species predicted a total of 166 proteins containing Sec-dependent signal peptides in the CLas strain psy62, from which 86 have been already experimentally validated [134]. These are potential effector proteins, and 106 of them share common homologues with Las_Ishi-1 and Las_gxpsy. But only 45 Secdependent proteins were shared among HLB-associated Ca. Liberibacter species (CLas, CLam and CLaf) [135, 136]. The detection of transcripts of the general secretion system provides further support for the participation of the detected Sec-dependent proteins in the late process of infection of the gut epithelium of $D$. citri. The Sec pathway secretion system transports proteins involved in bacterial cell functions and survival [133]. The Sec pathway is represented by two independent pathways, both detected in the gut of $\mathrm{CLas}^{+}$psyllids: the post-translational pathway represented by the expression of SecA (DN14962_c0_g1_i1) and SecY (DN15568_c0_ g3_i3); and the co-translational pathway represented by the signal recognition particle receptor FtsY (DN1686 c0_g1_i1) [137]. We did not detect SecB expression in the gut of adult psyllids, indicating SecA can also act as a chaperone complementing the required activity of $\mathrm{SecB}$ as reported in Escherichia coli [138].

Combined ultrastructure, proteomics and transcriptomics analysis of the gut epithelium of CLas-infected psyllids suggested CLas acquisition into the hemocoel would rely on the programmed cell death of CLas-infected epithelial gut cells, although CLas infection has little impact on the fitness of adult psyllids [19]. The low fitness impact of CLas infection to adult D. citri indicates CLas acquisition into the hemocoel would occur through an exocytosis process, as suggested for the $\mathrm{Ca}$. Liberibacter solanacearum vectored by potato psyllids [139].

By following the proposed subroutines based on mechanistic and essential aspects of cell death proposed by the Nomenclature Committee on Cell Death [140], our 
transcriptomic analysis could not support the proposition that CLas-infected epithelial gut cells of psyllids would initiate the process of programmed cell death (PCD) as proposed by Kruse et al. [19]. We did not observe transcription of caspases, a very common protein to several subroutines of cell death, as well as other aspects that would allow this characterization [140]. Yet, we were unable to confidently characterize one of the subroutines of cell death described when analyzing the transcriptional profile of $\mathrm{CLas}^{+}$psyllids, once the molecular mechanisms involved were not all represented. However, we can confidently report the activation/inhibition of the expression of genes involved in processes of regulated cell death. The high expression of the lysosomal cathepsin L1 (DN15698_c4_g11_i2) in A2CLas ${ }^{+}$ psyllids would support the activation of lysosomedependent cell death, which requires intracellular perturbations to permeabilize the lysosomal membrane to result in the release of cytosolic cathepsins. Mitochondrial outer membrane permeabilization and caspases are not necessarily required for this process of cell death, and lysosome-dependent cell death is an important response to pathophysiological conditions induced by intracellular pathogens [140].

The overexpression of the psyllid BCL2/adenovirus E1B $19 \mathrm{kDa}$ protein-interacting protein 3 (BNIP3) (DN 20168_c2_g3_i4) in $\mathrm{A3CLas}^{+}$also supports the gut epithelium of $\mathrm{CLas}^{+}$psyllids go into lysosome-dependent cell death. BNIP3 are pro-apoptotic proteins that open the pores of mitochondrial outer membrane, resulting in mitochondria dysregulation due the loss of membrane potential and ROS production in mitochondria [141, 142]. But BNIP3 oligomerization with BCL2 has been demonstarted not to be required for cell death [141]. Besides, the down-regulation of the psyllid senescence-associated protein transcript (DN16041_c1_g1_i2) in the gut of A3CLas ${ }^{+}$ psyllids demonstrates CLas suppresses cell senescence and the further dysfunctional growth of the epithelial cells due the activation of senescence-associated secretory phenotype $[143,144]$. Cellular senescence is not considered a form of regulated cell death [140].

The differential expression of a putative juvenile hormone binding protein (JHBP) (DN21423_c1_g12_i2; DN21423_c1_g12_i1) in $\mathrm{CLas}^{+}$psyllids (down-regulation in the gut of $\mathrm{A2CLas}^{+}$; up-regulation in $\mathrm{A3CLas}^{+}$) demonstrates the epithelial cells count with different levels of hormonal stimulation. JHBPs are proteins that act as shuttles for juvenile hormone $(\mathrm{JH})$ in the hemolymph, avoiding $\mathrm{JH}$ degradation by $\mathrm{JH}$-esterases and $\mathrm{JH}$-epoxide hydrolases [145]. JH is produced and released primarily by neurosecretory cells of the corpora allata of the central nervous system, and the availability of JHBP in the gut, if its activity in the gut is the same played in the hemolymph, indicates $\mathrm{JH}$ is alo available in the gut epithelial cells. JH has recently been shown to be produced by intestinal stem cells and enteroblasts of the gut epithelium of D. melanogaster, and to regulate cell growth and survival. The local JH activity was also shown relevant for damage response by gut cells, playing important roles in gut homeostasis [146]. If the pattern of expression of JHBP correlates with the availability of $\mathrm{JH}$ we can argue that at the same time CLas acts on the regulation of the gut epithelium by suppressing cell senescence, it can also induce the proliferation of stem cells in order to replace cells that were damaged due the release of CLas cell into the hemocoel and/or entered the lysosome-mediated cell death as discussed earlier.

The observed up-regulation of the transcriptional levels of nocturnin (DN19927_c10_g1_i6) in the gut of A2CLas ${ }^{+}$psyllids suggests that this circadian rhythm effector protein may be acting together with $\mathrm{JH}$ in regulating gut genes, just as the joint action of the circadian genes and $\mathrm{JH}$ in the regulation of genes acting on the transitional states of Pyrrhocoris apterus adults to reproductive diapause or not [147]. Nocturnin is classified as a deadenylase acting on the catalysis of poly(A) tail of target mRNAs and/or targeting noncoding RNAs, and has been implicated in metabolic regulation, development and differentiation [148]. But recent studies with curled, the nocturnin ortholog in Drosophila proved nocturnin is an $\operatorname{NADP}(\mathrm{H}) 2^{\prime}$-phosphatase acting on the conversion of the dinucleotide $\mathrm{NADP}^{+}$into $\mathrm{NAD}^{+}$and $\mathrm{NADPH}$ into $\mathrm{NADH}$, regulating mitochondrial activity and cellular metabolism in response to circadian clock [149]. NADP(H)2'-phosphatase activity of nocturnin was later reported in vertebrates, with the demonstration of the colocalization of nocturnin in mitochondria, cytosol and endoplasmic reticulum-bound pools depending on the isoform [150]. Thus, nocturnin acts as a regulator of the intracellular levels of $\mathrm{NADP}(\mathrm{H})$ and the oxidative stress response. The role of nocturnin in the regulation of cell metabolism and the oxidative stress response supports the required energy supply to sustain the increased gene expression activity in $\mathrm{CLas}^{+}$gut epithelium cells.

The expression profile of CLas infecting the gut epithelium of $\mathrm{A3CLas}^{+}$psyllids demonstrates CLas has an increased protein synthesis activity as expected by the overexpression of DEAD/DEAH box helicase (DN4052 c0_g1_i1), a protein involved in ribosome biogenesis, RNA turnover and translation initiation [151]. Increased protein activity is also supported by the increased expression of molecular chaperones, such as the trigger factor (DN13945_c0_g1_i1), GroEL (DN14757_c0_g1_i1) and DnaK (DN15220_c0_g1_i1), which prevent protein misfolding and aggregation [152, 153].

\section{CLas multiprotein complexes}

The protein HlyD family efflux transporter periplasmic adaptor subunit (DN11766_c0_g1_i1) and the outer 
membrane factor translocation protein TolB (DN15087_ c0_g1_i3) are essential components for functioning the pump of tripartite efflux systems. HlyD connects primary and secondary inner membrane transporters to the outer membrane factor TolB. CLas expressed several inner membrane transporters belonging to the MSF and $\mathrm{ABC}$ families. TolB has been shown to interact with a range of proteins including cell-killing proteins [154, 155]. In Xylella fastidiosa TolB was shown to be involved in biofilm development as one of the members of the Tol - Pal system. TolB associates with other proteins than $\mathrm{Pal}$ in the outer membrane and periplasm, aiding $X$. fastidiosa in maintaining membrane integrity [156].

\section{Additional CLas transcriptional regulators}

The detection of several other uncharacterized response regulators (DN10585_c0_g1_i1; DN10585_c0_g2_i1; DN12200_c0_g1_i1; DN58285_c0_g1_i1) and transcriptional regulators (DN11066_c0_g1_i1; DN15145_c0_g1_ i2; DN15145_c0_g1_i5) indicates other CLas twocomponent systems are also being activated by environmental stimuli, such as the two-component system sensor histidine kinase AtoS (DN14305_c0_g2_i1; DN15701_c0_g1_i1). The two-component system sensor histidine kinase AtoS is a member of the AtoS/AtoC regulatory system, but we did not identify the AtoC response regulator in the CLas transcriptome. This regulatory system is better known by the induction of AtoS by acetoacetate. AtoS then phosphorylates AtoC that will in turn stimulate the expression of the atoDAEB operon for the catabolism of short chain fatty acids. We could not reliably identify transcripts belonging to the ato$D A E B$ operon to demonstrate CLas requirements for short chain fatty acids. We propose that the activation of the AtoS/AtoC regulatory system is instead acting on the regulation of the flagellar regulon, regulating the expression of CLas genes involved in cell motility and chemotaxis, as reported for Escherichia coli in response to acetoacetate or spermidine [157].

\section{Cross-talk on nutrient deficiency}

The high expression of sulfonate $\mathrm{ABC}$ transporter permease (DN15690_c0_g1_i2) in all $\mathrm{CLas}^{+}$psyllids suggests CLas signals the host to supply its requirements for sulfur. Sulfur is a ubiquitous element involved in a number of different processes in organisms [158]. The sulfonate $\mathrm{ABC}$ transporter permease is involved in sulfur/sulfonate uptake, and the higher expression observed in $\mathrm{A3CLas}^{+}$as compared to A1CLas ${ }^{+}$points for an increased demand late in the infection process.

Sulfur is also used in the biogenesis of Fe-S clusters, which are produced in conditions of oxidative stress and iron deprivation. The biogenesis of Fe-S clusters was observed by the expression of two unclassified cysteine sulfurases (DN12917_c0_g1_i1 and DN35541_c0_g1_i1), the cysteine desulfuration protein SufE (DN1144_c0_g1_ i1), the Fe-S cluster assembly protein SulfB (DN14252 c0_g1_i1), and the iron-sulfur cluster carrier protein ApbC (DN17762_c4_g2_i3) [159, 160]. All four genes had an increased expression in $\mathrm{ABCLas}^{+}$as compared to A1CLas ${ }^{+}$. Sulfur requirements by CLas could also be used for protection against oxidative stress as demonstrated by the expression of the thioredoxin-dependent thiol peroxidase (DN10526_c0_g1_i1) [161, 162].

The high expression of CLas phosphate $\mathrm{ABC}$ transporter permease subunit PstC (DN40256_c0_g1_i1; DN67283_c0_g1_i1), putative two-component sensor histidine kinase transcriptional regulatory protein (DN14305_c0_g2_i1), two-component sensor histidine kinase (PhoR) (DN28075_c0_g1_i1; DN67626_c0_g1_i1), two component response regulator protein (PhoB) (DN14869_c0_g1_i1), alkaline phosphatase (DN12828_ c0_g1_i2; DN39297_c0_g1_i2) and NTP pyrophosphohydrolase (DN2128_c0_g1_i1) demonstrates CLas cells are exposed to phosphate restriction while infecting the gut epithelium of adult psyllids. Phosphate is generally the major source of phosphorus to bacteria, a vital nutrient for living organisms. Phosphorus is important in several processes (energy metabolism, intracellular signaling, among others). Misregulation of phosphorus availability in bacteria is sensed by the PhoB/PhoR two-component regulatory system, leading to the activation of the Pho regulon. The Pho regulon regulates the expression of other genes, producing phenotypes that can differ in morphology and virulence in response to phosphate deprivation [163, 164]. Thus, the expression of both members of the PhoB/PhoR two component regulatory system and the phosphate $\mathrm{ABC}$ transporter permease subunit PstC, that is in charge to transport extracellular phosphate to the cytosol, proves CLas was exposed to phosphate concentrations below $4 \mu \mathrm{M}$, a threshold that generally turns on the expression of the response regulator PhoB. Furthermore, the expression of alkaline phosphatase and NTP pyrophosphohydrolase, which both act on phosphorus-containing substrates [163] demonstrates CLas increased the catabolism of substrates capable of releasing phosphorus nutrient.

The requirement of CLas for phosphate is also demonstrated by the up-regulation of the psyllid glycerophosphocholine phosphodiesterase GPCPD1 gene, as observed by the increased abundance of its transcript (DN15741_c0_g2_i2) in A3CLas ${ }^{+}$. The contribution of GPCPD1 in phosphate production results from the downstream processing of GPCPD1 hydrolysis products of glycerophosphocholine, choline and glycerolphosphate. At the same time glycerophosphocholine hydrolysis can provide phosphates for cell metabolism, the choline can serve as as substrate for phosphatidylcholine 
synthesis. Phosphatidylcholine is a major lipid component of membranes of eukaryotes, although several bacterial symbionts and pathogens carry phosphatidylcholine synthases, showing their requirement for this lipid component of cell membranes, including those belonging to Rhizobiaceae as $\mathrm{Ca}$. Liberibacter. Choline serves as substrate for the osmoprotectant glycine betaine synthesis and as an energy substrate to support cell growth in Rhizobiaceae $[165,166]$.

The role of host-produced choline in CLas metabolism is supported by the up-regulation of CLas glycine/betaine $A B C$ transporter substrate-binding protein (DN15178_c0_g1_i1) in the gut of $A 3 C L a s^{+}$. ABC transporters were reported to have several important roles in $\mathrm{Ca}$. Liberibacter asiaticus, from the importation of nutrients like choline to the exportation of virulence factors [167]. Thus, we believe the increased activity of GPCP D1 in $\mathrm{A3CLas}^{+}$is a result of the recycling of glycerophosphocholine for choline utilization in the recovery of membranes of the gut epithelial cells of the host psyllid as a response to cell infection and/or in the supplementation of choline to CLas metabolism.

CLas is known to induce profound physiological changes in citrus plants, affecting the nutritional content of CLas-infected plants to psyllids, which have in turn altered transcriptional profiles and protein abundance when feeding on CLas-infected and health citrus plants $[24,107]$. Therefore, it is impossible to distinguish if the indication of nutritional restriction CLas encounters when infecting the gut epithelium of psyllids is an indirect effect of the host plant on the psyllids or if this is a direct response of psyllids to avoid CLas infection.

\section{Conclusion}

We conclude that CLas basically express its full arsenal of genes early in the invasion process of the gut of adults of $D$. citri. CLas survival in the gut of psyllids involves the regulation of ROS production and availability in the psyllid gut, and consequently the inhibition of activation ROS-dependent nitric oxide production and nitric oxide-dependent antimicrobial peptide synthesis. Our data suggests that gene expression regulation of adult psyllids is based on the up-regulation of proteins involved in alternative splice site selection. CLas regulates the peristalsis of the gut of the host and highly expresses genes involved in adherence proteins and biofilm formation to colonize the gut lumen. Our analysis also indicates the gut epithelial cells are invaded by CLas by a process mediated by the down-regulation of leptin receptor and its internalization in early endosomes in the psyllid gut cells. This process is followed by the regulation of several host genes that lead to vesicles containing CLas, which includes the suppression of the cell machinery involved in the process of cell senescence. The intense cross talk between CLas and the host cells is mediated by a high number of candidate effector proteins we identified. These proteins are potential targets for the development of management strategies directed to interfere with essential metabolic pathways to invasion, infection and multiplication of CLas in the vector D. citri.

\section{Material and Methods Diaphorina citri rearing and plants}

A colony of CLas-free Asian Citrus Psyllid (ACP) was initiated with insects collected from Murraya paniculata (L.) Jack, syn. Murraya exotica L. (Sapindales: Rutaceae) in the state of São Paulo, Brazil in 2009.

\section{Diaphorina citri gut collection and RNA extraction}

Adults of D. citri (7 to 10 days after emergence) were transferred to uninfected and CLas-infected citrus plants [Citrus x sinensis (L.) Osbeck, grafted in 'Rangpur' lime (C. x limonia Osbeck)] for an exposure period (EP) of 1 , 2, 3, 4, 5 and 6 days. For each exposure period, three biological replicates were collected (1 replicate $=100$ individuals). Third-instars of $D$. citri were also transferred to CLas-infected and uninfected (control) citrus plants for collection of RNA and differential transcriptional analysis. Nymphs were allowed to feed for 4 days on CLas-infected and control plants. Afterwards, three biological replicates/treatment $(1$ replicate $=200$ nymphs $)$ were collected and stored in RNALater (Invitrogen/ ThermoFisher Scientific, Waltham, MA, United States, USA) for further processing and analysis.

After each exposure time, adults were collected and the gut dissected under aseptic conditions. A similar procedure was used for nymphs, but in this case insects we opted by extracting RNA from whole nymphs, as initial attempts to dissect the suitable gut samples for downstream analysis were very time-consuming and had a low success rate.

The obtained guts/nymphs were stored in RNALater (Invitrogen/ThermoFisher Scientific, Waltham, MA, USA) at $-80^{\circ} \mathrm{C}$ until RNA extraction. Total RNA extraction was performed using the SV RNA Isolation System Kit (Promega Madison, WI, USA), following the manufacturers' recommendations. Samples were lysed in 175 $\mu \mathrm{L}$ of RNA lysis buffer added with $\beta$-mercaptoethanol and $350 \mu \mathrm{L}$ of RNA dilution buffer for tissue disruption using a TissueLyser II LT ${ }^{\mathrm{TM}}$ (Qiagen, Hilden, Germany). Samples were incubated at $70^{\circ} \mathrm{C}$ for $3 \mathrm{~min}$, centrifuged $\left(12,000 \mathrm{~g} \times 10 \mathrm{~min} \times 4^{\circ} \mathrm{C}\right)$, trapped in a column and washed with $95 \%$ ethanol by centrifugation $(12,000 \mathrm{~g} \times$ $10 \mathrm{~min} \times 4^{\circ} \mathrm{C}$ ). Total RNA was recovered in $350 \mu \mathrm{L}$ of RNA wash solution following centrifugation $(12,000 \mathrm{~g} \times$ $1 \min \times 4^{\circ} \mathrm{C}$ ). Afterwards, samples were treated with 50 $\mu \mathrm{L}$ of DNAse mix ( $40 \mu \mathrm{L}$ buffer 'yellow core' $+5 \mu \mathrm{L} 0.09$ $\mathrm{M} \mathrm{MnCl}_{2}+5 \mu \mathrm{L}$ DNAse I) for $15 \mathrm{~min}$ at room 
temperature. Samples were added with $200 \mu \mathrm{L}$ of DNAse stop solution and subjected to centrifugation $(13,000 \mathrm{~g} \times$ $1 \mathrm{~min} \times 4^{\circ} \mathrm{C}$ ). Samples were washed in $600 \mu \mathrm{L}$ cleaning solution followed by a second wash in $250 \mu \mathrm{L}$ following centrifugation $\left(12,000 \mathrm{~g} \times 1 \mathrm{~min} \times 4^{\circ} \mathrm{C}\right)$. The pelleted RNA was recovered in $30 \mu \mathrm{L}$ of nuclease-free water, and RNA concentration verified using NanoDrop V 3.8.1 (ThermoFischer).

RNA samples containing residual DNA contaminants were further treated with $2 \mu \mathrm{L}$ of buffer $(10 \times)$ and $2 \mu \mathrm{L}$ of Turbo ${ }^{\text {TM }}$ DNAse (2 U/ $\mu$ l) (Ambion/ThermoFisher Scientific, Vilnius Lithuania). Samples were incubated at $37^{\circ} \mathrm{C}$ for $30 \mathrm{~min}$ followed by DNAse inactivation by adding $2 \mu \mathrm{L}$ of DNAse Inactivation Reagent (Ambion/ThermoFisher Scientific, Vilnius Lithuania). After $5 \mathrm{~min}$ at room temperature, samples were centrifuged $(13,000 \mathrm{~g} \times$ $1.5 \mathrm{~min} \times 4^{\circ} \mathrm{C}$ ) and the supernatant collected and stored at $-80^{\circ} \mathrm{C}$. DNA elimination was confirmed by testing the amplification of the wingless gene ( $w g)$ of ACP [168].

All plants and insects were subjected to quantitative PCR analysis for verification of CLas infection using the TaqMan qPCR Master Mix Kit (Ambion/ThermoFisher Scientific, Vilnius Lithuania) [169]. In insects, the average $\mathrm{Ct}$ value found for the $w g$ gene in the libraries was 30.7 , while the average $\mathrm{Ct}$ value in plants for the $16 \mathrm{~S}$ rRNA gene from CLas was 29.6. Samples with a Ct value under 35 were considered positive for CLas.

After confirmation of the Ca. L. asiaticus infection status of each sample, RNA obtained from the gut samples of adults were pooled in equimolar concentrations to yield three exposure periods: i) A1CLas: adults that fed on healthy citrus plant ( $\mathrm{CLas}^{-}$) for 1-2 days; ii) A2CLas: for 3-4 days; iii) A3CLas: for 5-6 days; iv) A1CLas ${ }^{+}$: adults that fed on infected citrus plant $\left(\mathrm{CLas}^{+}\right)$for 1-2 days; v) $\mathrm{A2CLas}^{+}$: for 3-4 days; and vi) $\mathrm{ABCLas}^{+}$: for 5-6 days. In the case of the whole nymphs, two samples were produced: N1CLas ${ }^{+}$: for nymphs after 4 days of feeding on CLas-infected citrus plants, and N1CLas: for nymphs after 4 days of feeding on control plants.

Samples were subjected to mRNA enrichment through eukaryote and prokaryote rRNA removal using the RiboZero rRNA Removal Epidemiology Kit (Illumina, San Diego, CA, USA), following the manufacturers' instructions. RNA integrity was confirmed using the Agilent Bioanalyzer 1000 (Agilent Technologies, Santa Clara, CA, USA).

\section{Library preparation and sequencing}

cDNA libraries were prepared for sequencing using the cDNA TruSeq RNA Library Prep Kit (Illumina, San Diego, CA, USA) following a paired-end $(2 \times 100 \mathrm{bp})$ strategy. The cDNA produced was end-repaired and adenosine was added at the 3 ' end of each cDNA fragment to guide the ligation of specific adapters. Adapters consisted of primers for transcription and a specific index to code each sample. Samples were enriched with limited-cycle PCR and analyzed to confirm the success of sample preparation before sequencing using the Illumina HiScanSQ platform (Illumina, San Diego, CA, USA) available at the Multiusers Center of Agricultural Biotechnology at the Department of Animal Sciences, ESALQ/USP.

\section{De novo transcriptome assembly}

Reads quality were visualized in FastQC software [170] before adapters removal and quality filtering by trimming the leading (LEADING:3) and trailing (TRAILING: 3) nucleotides until the quality was higher than 3 , and then using a sliding window of 4 nucleotides and trimming when scores were lower than 22 (SLIDINGWINDOW 4:22). Quality filtering was done using Trimmomatic-0.36 [171].

All reads obtained were used to assemble a de novo transcriptome using the pipeline available in the Trinityv.2.4.0 software [172]. Both paired and unpaired trimmed and quality-filtered reads were used to assemble the de novo transcriptome, which was further used as the reference transcriptome for the RNA-Seq experiments. Assemblage was obtained using normalization of the reads coverage $(<50)$ and the minimum contig size selected was 200 nucleotides.

The transcripts obtained were functionally annotated using the BlastX algorithm for putative identification of homologous sequences, with an $e$-value cut-off $<10^{-3}$. Annotated sequences were curated and grouped into categories according to their function using Blast2 $\mathrm{Go}^{\circ}$ [173] with an $e$-value cut-off $<10^{-6}$ and EggNOGmapper 4.5.1 [174]. Transcripts putatively identified as belonging to insects and $\mathrm{Ca}$. L. asiaticus were checked against the KEGG database (Kyoto Encyclopedia of Genes and Genomes) [175] to verify the metabolic pathways represented in the obtained de novo transcriptome. Transcripts of Diaphorina citri, Ca. Liberibacter spp. and Wolbachia spp. were filtered using Blast2Go version Pro [176].

\section{Differential gene expression analyses}

Changes in the pattern of gene expression were evaluated separately in gut of ACP adults infected or not by $\mathrm{Ca}$. L. asiaticus using the CLC Genomics Workbench 20.0 software (QIAGEN, Aarhus, Denmark). Reads from each library were counted against the de novo transcriptome, and counts were normalized as transcripts per million reads (TPM). TPM values for each sample were used to calculate fold-change ratios for comparative analyses of the gene expression of control (CLas ${ }^{-}$) versus CLas-infected insects $\left(\mathrm{CLas}^{+}\right)$within each feeding interval (1-2 d, 3-4 d, 5-6 d). Only the transcript that were 
counted in two of the replicates of a particular treatment were further taken for comparative analysis. Data were analyzed using multifactorial statistics based on a negative binomial Generalized Linear Model (GLM). The values of fold change obtained were corrected with False Discovery Rate (FDR) method and only transcripts that showed $p$-value $\leq 0.05$ and log fold change $>|2|$ between treatments were considered differentially expressed.

\section{Abbreviations}

A1CLas: adults that fed on healthy citrus plant for 1-2 days; A2CLas: adults that fed on healthy citrus plant for 3-4 days; A3CLas: adults that fed on healthy citrus plant for 5-6 days; A1CLas ${ }^{+}$adults of Asian Citrus Psyllid that fed on CLas-infected citrus plant for 1-2 days; A2CLas $^{+}$: adults of Asian Citrus Psyllid that fed on CLas-infected citrus plant for 3-4 days; A3CLas ${ }^{+}$: adults of Asian Citrus Psyllid that fed on Clas-infected citrus plant for 5-6 days; ACP: Asian Citrus Psyllid; ADK: Adipokinetic Hormone; AMP: Antimicrobial Peptides; BLAST: Basic Local Alignment Search Tool; BNIP3: BCL2/Adenovirus E1B 19 Kda Protein-Interacting Protein 3; CLaf: Candidatus Liberibacter africanus; CLam: Candidatus Liberibacter americanus; CLas: Candidatus Liberibacter asiaticus; CLas: adults of Asian Citrus Psyllid that fed on healthy citrus plant; Clas ${ }^{+}$: adults of Asian Citrus Psyllid that fed on CLas-infected citrus plant; DE: Differential Expression; ER: Endoplasmic Reticulum; FC: Fold Change; FDR: False Discovery Rate; GLM: Generalized Linear Model; HLB: Huanglongbing; JH: Juvenile Hormone; JHBP: Juvenile Hormone Binding Protein; KEGG: Kyoto Encyclopedia of Genes and Genomes; N1CLas: Nymphs of Asian Citrus Psyllid fed for 4 days on healthy citrus plants;

N1CLas ${ }^{+}$: Nymphs of Asian Citrus Psyllid fed for 4 days of feeding on CLasinfected citrus plants; PhoB: Two-component Response Regulator Protein; PhoR: Two-component Sensor Histidine Kinase; PPI: Peptidyl-Prolyl Isomerase; PPO: Prophenoloxidases; RBOH: Respiratory Burst Oxidase Homologs; RNS: Reactive Nitrogen Species; ROS: Reactive Oxygen Species; SNAP: Soluble NSF Attachment Protein; TPM: Transcripts Per Million Reads;

TPR: Tetratricopeptide Repeat

\section{Supplementary Information}

The online version contains supplementary material available at https://doi. org/10.1186/s12864-021-07988-2

Additional file 1: The analysis of the 248,850 contigs was conducted by Blast2Go, using Blastx to recover annotations with significant homology from the NCBI. All terms 'Biological Processes', 'Molecular Function' and 'Cellular Component' at level 2 are represented as percent over total number of sequences.

Additional file 2: Distribution of alignments and transcripts of de novo assembly of Diaphorina citri gut that fed on health and CLas-infected citrus plant, after search for similarity in NCBI databank.

Additional file 3: Differentially expressed CLas genes in the gut of Diaphorina citri adults after 1-2 d (A1Clas+), 3-4 d (A2Clas+) and 5-6 d (A3Clas+) of infection by feeding on CLas-infected citrus plants.

\section{Acknowledgements}

Not applicable.

\section{Authors' contributions}

FLC and NAW designed the experiments. FLC, NAW and LP secured the funds. JCD collected insect samples and extracted RNA. BLM processed the sequencing data and assembled the de novo transcriptome. FMMB analyzed the data. FMMB and FLC wrote the paper. All authors read and approved the final manuscript.

\section{Funding}

FAPESP provided the post-doctoral fellowship to FMMB (grant number 2018/ 24234-4) and BLM (grant number 2018/24768-9), and a research grant to FLC, NAW and LP to support this research (grant number 2015/07011-3). CNPq provided the PhD fellowship to JCD (grant number 141042/2017-6).

\section{Availability of data and materials}

All cDNA libraries produced from RNA obtained from samples analyzed are fully available as SRA files through NCBI (https://www.ncbi.nlm.nih.gov/ bioproject/) under BioProject ID PRJNA722422. All remaining data generated or analysed during this study are included in this published article and its additional information files.

\section{Declarations}

Ethics approval and consent to participate

This study did not require permits for seeds, plant and/or animal collection, and maintenance under laboratory conditions. This study complies with institutional, national and international guidelines and legislation.

\section{Consent for publication}

Not applicable.

\section{Competing interests}

The authors declare that they have no competing interests.

\section{Author details}

${ }^{1}$ Insect Interactions Laboratory, Department of Entomology and Acarology, Luiz de Queiroz College of Agriculture, University of São Paulo, Avenida Pádua Dias 11, Piracicaba, São Paulo 13418-900, Brazil. ${ }^{2}$ Fund for Citrus Protection (FUNDECITRUS), Araraquara, São Paulo 14807-040, Brazil. Institute of Chemistry, São Paulo State University - UNESP, Araraquara, São Paulo, Brazil. ${ }^{4}$ Instituto de Biología Molecular y Celular de Plantas (IBMCP), Consejo Superior de Investigaciones Científicas (CSIC), Universidad Politécnica de Valencia (UPV), 46022 Valencia, Spain.

Received: 16 April 2021 Accepted: 3 September 2021

Published online: 21 September 2021

\section{References}

1. Huang W, Reyes-Caldas P, Mann M, Seifbarghi S, Kahn A, Almeida RPP, et al. Bacterial vector-borne plant diseases: unanswered questions and future directions. Mol Plant. 2020;13(10):1379-93. https://doi.org/10.1016/j.molp.202 0.08.010.

2. Perilla-Henao LM, Casteel CL. Vector-borne bacterial plant pathogens: interactions with hemipteran insects and plants. Front Plant Sci. 2016 Aug 9; 7:1163. https://doi.org/10.3389/fpls.2016.01163.

3. Mauck KE, Chesnais Q, Shapiro LR. Evolutionary determinants of host and vector manipulation by plant viruses. Adv Virus Res. 2018;101:189-250. https://doi.org/10.1016/bs.aivir.2018.02.007.

4. Bové JM. Huanglongbing: A destructive newly emerging, century-old disease of citrus. J Plant Pathol. 2006;88:7-37. https://doi.org/10.4454/jpp. v88i1.828.

5. Graça JV, Douhan GW, Halbert SE, Keremane ML, Lee RF, Vidalakis G, et al. Huanglongbing: An overview of a complex pathosystem ravaging the world's citrus. J Integr Plant Biol. 2016;58(4):373-87. https://doi.org/10.1111/ jipb.12437.

6. Tomaseto AF, Marques RN, Fereres A, Zanardi OZ, Volpe HXL, Alquézar B, et al. Orange jasmine as a trap crop to control Diaphorina citri. Sci Rep. 2019;9(1):2070. https://doi.org/10.1038/s41598-019-38597-5.

7. Bassanezi RB, Lopes AS, Miranda MP, Wulff NA, Volpe HXL, Ayres AJ Overview of citrus huanglongbing spread and management strategies in Brazil. Trop Plant Pathol. 2020;45:251-64. https://doi.org/10.1007/s40858-02000343-y.

8. Abebe E, Gugsa G, Ahmed M. Review on major food-borne zoonotic bacterial pathogens. J Trop Med. 2020;4674235. https://doi.org/10.1155/202 0/4674235.

9. Dala-Paula BM, Plotto A, Bai J, Manthey JA, Baldwin EA, Ferrarezi RS, et al. Effect of huanglongbing or greening disease on orange juice quality, a review. Front Plant Sci. 2019;9:1976. https://doi.org/10.3389/fpls.2018.01976.

10. Singerman A, Rogers ME. The economic challenges of dealing with citrus greening: the case of Florida. J Integr Pest Manag. 2020;1 1(1):1-7. doi: https://doi.org/10.1093/jipm/pmz037.

11. CABI/EPPO. Candidatus Liberibacter asiaticus [Distribution map]. 4th ed. Wallingford: CABI.; 2017.

12. Ajene IJ, Khamis FM, van Asch B, Pietersen G, Seid N, Rwomushana I, et al. Distribution of Candidatus Liberibacter species in Eastern Africa, and the first 
report of Candidatus Liberibacter asiaticus in Kenya. Sci Rep. 2020;10:3919. https://doi.org/10.1038/s41598-020-60712-0.

13. Wulff NA, Daniel B, Sassi RS, Moreira AS, Bassanezi RB, Sala I, et al. Incidence of Diaphorina citri carrying Candidatus Liberibacter asiaticus in Brazil's citrus belt. Insects. 2020;11(10):672. https://doi.org/10.3390/insects11100672.

14. Jagoueix S, Bove JM, Garnier M. The phloem-limited bacterium of greening disease of citrus is a member of the alpha subdivision of the Proteobacteria. Int J Syst Bacteriol. 1994;44(3):379-786. https://doi.org/10.1099/002 07713-44-3-379.

15. Bové JM, Garnier M. Phloem-and xylem-restricted plant pathogenic bacteria. Plant Sci. 2003;164:423-38. https://doi.org/10.1016/S0168-9452(03)00033-5.

16. Bendix C, Lewis JD. The enemy within: phloem-limited pathogens. Mol Plant Pathol. 2018;19(1):238-54. https://doi.org/10.1111/mpp.12526.

17. Ammar E-D, Shatters RG Jr, Hall DG. Localization of Candidatus Liberibacter asiaticus, associated with citrus Huanglongbing disease, in its psyllid vector using fluorescence in situ hybridization. J Phytopathol. 2011;159:726-34. https://doi.org/10.1111/j.1439-0434.2011.01836.x

18. Ammar E-D, Hall DG, Shatters RG Jr. Ultrastructure of the salivary glands, alimentary canal and bacteria-like organisms in the Asian citrus psyllid, vector of citrus Huanglongbing disease bacteria. J Microsc Ultrastruct. 2017; 5:9-20. https://doi.org/10.1016/j.jmau.2016.01.005.

19. Kruse A, Fattah-Hosseini S, Saha S, Johnson R, Warwick E, Sturgeon K, et al. Combining 'omics and microscopy to visualize interactions between the Asian citrus psyllid vector and the Huanglongbing pathogen Candidatus Liberibacter asiaticus in the insect gut. PLoS ONE. 2017;12(6):e0179531. https://doi.org/10.1371/journal.pone.0179531.

20. Ammar E-D, Achor D, Levy A. Immuno-ultrastructural localization and putative multiplication sites of Huanglongbing bacterium in Asian Citrus Psyllid Diaphorina citri. Insects. 2019;10(12):422. https://doi.org/10.3390/ insects10120422.

21. Gray S, Cilia M, Ghanim M. Circulative, "nonpropagative" virus transmission: an orchestra of virus-, insect-, and plant-derived instruments. Adv Virus Res. 2014;89:141-99. https://doi.org/10.1016/B978-0-12-800172-1.00004-5.

22. Ammar E-D, Ramos JE, Hall DG, Dawson WO, Shatters RG Jr. Acquisition, replication and inoculation of Candidatus Liberibacter asiaticus following various acquisition periods on Huanglongbing-infected citrus by nymphs and adults of the Asian Citrus Psyllid. PLOS ONE. 2016;11(7):e0159594. https://doi.org/10.1371/journal.pone.0159594.

23. Martinelli AF, Uratsu SL, Albrecht U, Reagan RL, Phu MP, Britton M, et al. Transcriptome profiling of citrus fruit response to Huanglongbing disease. PLoS ONE. 2012;7:e38039. https://doi.org/10.1371/journal.pone.0038039.

24. Fu S, Shao J, Zhou C, Hartung JS. Transcriptome analysis of sweet orange trees infected with 'Candidatus Liberibacter asiaticus' and two strains of Citrus Tristeza Virus. BMC Genomics. 2016;17:349. https://doi.org/10.1186/s12 864-016-2663-9.

25. Hu Y, Zhong X, Liu X, Lou B, Zhou C, Wang X. Comparative transcriptome analysis unveils the tolerance mechanisms of Citrus hystrix in response to 'Candidatus Liberibacter asiaticus' infection. PLOS ONE. 2017;12:e0189229. https://doi.org/10.1371/journal.pone.0189229.

26. Ammar E, Shatters RG Jr, Lynch C, Hall DG. Detection and relative titer of Candidatus Liberibacter asiaticus in the salivary glands and alimentary canal of Diaphorina citri (Hemiptera: Psyllidae) vector of citrus Huanglongbing disease. Ann Entomol Soc Am. 2011;104(3):526-33. https://doi.org/10.1 603/AN10134.

27. Ammar E-D, George J, Sturgeon K, Stelinski LL, Shatters RG. Asian citrus psyllid adults inoculate Huanglongbing bacterium more efficiently than nymphs when this bacterium is acquired by early instar nymphs. Sci Rep. 2020;10(1):18244. https://doi.org/10.1038/s41598-020-75249-5.

28. Vyas M, Fisher TW, He R, Nelson W, Yin G, Cicero JM, et al. Asian citrus psyllid expression profiles suggest Candidatus Liberibacter asiaticusmediated alteration of adult nutrition and metabolism, and of nymphal development and immunity. PLoS ONE. 2015;10(6):e0130328. https://doi. org/10.1371/journal.pone.0130328.

29. Yu HZ, Li NY, Zeng XD, Song JC, Yu XD, Su HN, et al. Transcriptome analyses of Diaphorina citri midgut responses to Candidatus Liberibacter asiaticus infection. Insects. 2020;11(3):171. https://doi.org/10.3390/insects11 030171

30. Liu K, He J, Guan Z, Zhong M, Pang R, Han Q. Transcriptomic and metabolomic analyses of Diaphorina citri Kuwayama infected and noninfected with Candidatus Liberibacter asiaticus. Front Physiol. 2021;24(11): 630037. https://doi.org/10.3389/fphys.2020.630037.
31. Molki B, Thi Ha P, Mohamed A, Killiny N, Gang DR, Omsland A, et al. Physiochemical changes mediated by "Candidatus Liberibacter asiaticus" in Asian citrus psyllids. Sci Rep. 2019;9(1):16375. https://doi.org/10.1038/s41598019-52692-7.

32. Santos-Ortega Y, Killiny N. Silencing of sucrose hydrolase causes nymph mortality and disturbs adult osmotic homeostasis in Diaphorina citri (Hemiptera: Liviidae). Insect Biochem Mol Biol. 2018;101:131-43. https://doi. org/10.1016/j.ibmb.2018.09.003.

33. Lu ZJ, Huang YL, Yu HZ, Li NY, Xie YX, Zhang Q, et al. Silencing of the chitin synthase gene is lethal to the Asian citrus psyllid Diaphorina citri. Int J Mol Sci. 2019;20(15):3734. https://doi.org/10.3390/ijms20153734.

34. Lu ZJ, Zhou CH, Yu HZ, Huang YL, Liu YX, Xie YX, et al. Potential roles of insect tropomyosin1-X1 isoform in the process of Candidatus Liberibacter asiaticus infection of Diaphorina citri. J Insect Physiol. 2019;114:125-35. https://doi.org/10.1016/j.jinsphys.2019.02.012.

35. Yu X, Killiny N. RNA interference-mediated control of Asian citrus psyllid, the vector of the Huanglongbing bacterial pathogen. Trop Plant Pathol. 2020; 45:298-305. https://doi.org/10.1007/s40858-020-00356-7.

36. Lehane MJ, Billingsley PF. Biology of the insect midgut. Dordrecht: Springer Netherlands; 1996. 10.1007/978-94-009-1519-0.

37. Erlandson MA, Toprak U, Hegedus DD. Role of the peritrophic matrix in insect-pathogen interactions. J Insect Physiol. 2019;117:103894. https://doi. org/10.1016/j.jinsphys.2019.103894.

38. Buchon N, Broderick NA, Lemaitre B. Gut homeostasis in a microbial world: insights from Drosophila melanogaster. Nat Rev Microbiol. 2013;11(9):615-26. https://doi.org/10.1038/nrmicro3074.

39. Vallet-Gely I, Lemaitre B, Boccard F. Bacterial strategies to overcome insect defences. Nat Rev Microbiol. 2008;6(4):302-13. https://doi.org/10.1038/ nrmicro1870.

40. Perkins A, Nelson KJ, Parsonage D, Poole LB, Karplus PA. Peroxiredoxins: guardians against oxidative stress and modulators of peroxide signaling. Trends Biochem Sci. 2015;40(8):435-45. https://doi.org/10.1016/j.tibs.2015.05. 001.

41. Knoops B, Argyropoulou V, Becker S, Ferté L, Kuznetsova O. Multiple roles of peroxiredoxins in inflammation. Mol Cells. 2016;39(1):60-4. https://doi.org/1 $0.14348 /$ molcells.2016.2341.

42. Nelson K, Knutson ST, Soito L, Klomsiri C, Poole LB, Fetrow JS. Analysis of the peroxiredoxin family: using active-site structure and sequence information for global classification and residue analysis. Proteins. 2011; 79(3):947-64. https://doi.org/10.1002/prot.22936.

43. Jain M, Munoz-Bodnar A, Zhang S, Gabriel DW. A secreted 'Candidatus Liberibacter asiaticus' peroxiredoxin simultaneously suppresses both localized and systemic innate immune responses in planta. Mol Plant Microbe Interact. 2018;31(12):1312-22. https://doi.org/10.1094/MPMI-03-180068-R.

44. Kodrík D, Bednářová A, Zemanová M, Krishnan N. Hormonal regulation of response to oxidative stress in insects-an update. Int J Mol Sci. 2015;16(10): 25788-816. https://doi.org/10.3390/ijms161025788.

45. Turner AJ, Isaac RE, Coates D. The neprilysin (NEP) family of zinc metalloendopeptidases: genomics and function. Bioessays. 2001;23(3):261-9. https://doi.org/10.1002/1521-1878(200103)23:3<261::AID-BIES1036>3.0.CO;2-K

46. Shears SB, Hayakawa Y. Functional multiplicity of an insect cytokine family assists defense against environmental stress. Front Physiol. 2019;10:222. https://doi.org/10.3389/fphys.2019.00222.

47. Neish AS. Redox signaling mediated by the gut microbiota. Free Radic Res. 2013;47(11):950-7. https://doi.org/10.3109/10715762.2013.833331.

48. Wu SC, Liao CW, Pan RL, Juang JL. Infection-induced intestinal oxidative stress triggers organ-to-organ immunological communication in Drosophila. Cell Host Microbe. 2012;11(4):410-7. https://doi.org/10.1016/j.chom.2012.03.004

49. Jing T, Wang F, Qi F, Wang Z. Insect anal droplets contain diverse proteins related to gut homeostasis. BMC Genomics. 2018;19(1):784. https://doi.org/1 $0.1186 / s 12864-018-5182-z$

50. Melcarne C, Lemaitre B, Kurant E. Phagocytosis in Drosophila: From molecules and cellular machinery to physiology. Insect Biochem Mol Biol. 2019;109:1-12. https://doi.org/10.1016/j.ibmb.2019.04.002

51. Anderson KV. Toll signaling pathways in the innate immune response. Curr Opin Immunol. 2000;12(1):13-9. https://doi.org/10.1016/s0952-7915(99)0004 $5-x$

52. Nakhleh J, Moussawi LE, Osta MA. The melanization response in insect immunity. Adv In Insect Phys. 2017:52:83-109. https://doi.org/10.1016/bs.a iip.2016.11.002. 
53. Stokes BA, Yadav S, Shokal U, Smith LC, Eleftherianos I. Bacterial and fungal pattern recognition receptors in homologous innate signaling pathways of insects and mammals. Front Microbiol. 2015;6:19. https://doi.org/10.3389/ fmicb.2015.00019.

54. Brunner E, Peter O, Schweizer L, Basler K. pangolin encodes a Lef-1 homologue that acts downstream of armadillo to transduce the wingless signal in Drosophila. Nature. 1997;385(6619):829-33. https://doi.org/10.103 8/385829a0

55. Franz A, Shlyueva D, Brunner E, Stark A, Basler K. Probing the canonicity of the Wnt/Wingless signaling pathway. PLoS Genet. 2017;13(4):e1006700. https://doi.org/10.1371/journal.pgen.1006700.

56. Strand M, Micchelli CA. Quiescent gastric stem cells maintain the adult Drosophila stomach. Proc Natl Acad Sci U S A. 2011;108(43):17696-701. https://doi.org/10.1073/pnas.1109794108.

57. Cordero JB, Stefanatos RK, Scopelliti A, Vidal M, Sansom OJ. Inducible progenitorderived Wingless regulates adult midgut regeneration in Drosophila. EMBO J. 2012;31(19):3901-17. https://doi.org/10.1038/emboj.2012.248

58. Riddell CE, Lobaton Garces JD, Adams S, Barribeau SM, Twell D, Mallon EB. Differential gene expression and alternative splicing in insect immune specificity. BMC Genomics. 2014;15(1):1031. https://doi.org/10.1186/1471-21 64-15-1031.

59. Girard C, Will CL, Peng J, Makarov EM, Kastner B, Lemm I, et al. Posttranscriptional spliceosomes are retained in nuclear speckles until splicing completion. Nat Commun. 2012;3:994. https://doi.org/10.1038/ncomms1998.

60. Galganski L, Urbanek MO, Krzyzosiak WJ. Nuclear speckles: molecular organization, biological function and role in disease. Nucleic Acids Res. 2017;45(18):10350-68. https://doi.org/10.1093/nar/gkx759.

61. Bhattacharyya S, Feferman L, Tobacman JK. Arylsulfatase B regulates versican expression by galectin-3 and AP-1 mediated transcriptional effects. Oncogene. 2014;33(47):5467-76. https://doi.org/10.1038/onc.2013.483.

62. García B, Merayo-Lloves J, Martin C, Alcalde I, Quirós LM, Vazquez F. Surface Proteoglycans as mediators in bacterial pathogens infections. Front Microbiol. 2016;7:220. https://doi.org/10.3389/fmicb.2016.00220.

63. Roberts IS. The biochemistry and genetics of capsular polysaccharide production in bacteria. Annu Rev Microbiol. 1996;50:285-315. https://doi. org/10.1146/annurev.micro.50.1.285

64. Zhao M, Wang S, Li F, Dong D, Wu B. arylsulfatase b mediates the sulfonation-transport interplay in human embryonic kidney 293 cells overexpressing sulfotransferase 1A3. Drug Metab Dispos. 2016;44(9):1441-9. https://doi.org/10.1124/dmd.116.070938.

65. Massenti R, Lo Bianco R, Sandhu AK, Gu L, Sims C. Huanglongbing modifies quality components and flavonoid content of 'Valencia' oranges. J Sci Food Agric. 2016;96(1):73-8. https://doi.org/10.1002/jsfa.7061.

66. Dala-Paula BM, Raithore S, Manthey JA, Baldwin EA, Zhao W, et al. Active taste compounds in juice from oranges symptomatic for Huanglongbing (HLB) citrus greening disease. LWT - Food Science Technology. 2018;9:51825. https://doi.org/10.1016/j.lwt.2018.01.083.

67. Kiefl J, Kohlenberg B, Hartmann A, Obst K, Paetz S, Krammer G, et al. Investigation on key molecules of Huanglongbing (HLB)-induced orange juice off-flavor. J Agric Food Chem. 2018;66(10):2370-7. https://doi.org/10.1021/acs.jafc.7b00892.

68. Treutter D. Significance of flavonoids in plant resistance: a review. Environ Chem Lett. 2006;4:147-57. https://doi.org/10.1007/s10311-006-0068-8.

69. Hijaz FM, Manthey JA, Folimonova SY, Davis CL, Jones SE, Reyes-DeCorcuera J. An HPLC-MS characterization of the changes in sweet orange leaf metabolite profile following infection by the bacterial pathogen Candidatus Liberibacter asiaticus. PLoS ONE. 2013;8(11):e79485. https://doi. org/10.1371/journal.pone.0079485.

70. Hijaz F, Al-Rimawi F, Manthey JA, Killiny N. Phenolics, flavonoids and antioxidant capacities in Citrus species with different degree of tolerance to Huanglongbing. Plant Signal Behav. 2020;15(5):1752447. https://doi.org/10.1 080/15592324.2020.1752447.

71. Li Y, Park JS, Deng JH, Bai Y. Cytochrome c oxidase subunit IV is essential for assembly and respiratory function of the enzyme complex. J Bioenerg Biomembr. 2006b;38(5-6):283-91. https://doi.org/10.1007/s10863-006-9052-z.

72. Benguettat $O$, Jneid $R$, Soltys J, Loudhaief R, Brun-Barale A, Osman D, et al. The DH31/CGRP enteroendocrine peptide triggers intestinal contractions favoring the elimination of opportunistic bacteria. PLoS Pathog. 2018;14(9): e1007279. https://doi.org/10.1371/journal.ppat.1007279.

73. Hammer TJ, Janzen DH, Hallwachs W, Jaffe SP, Fierer N. Caterpillars lack a resident gut microbiome. Proc Natl Acad Sci U S A. 2017;114(36):9641-6. https://doi.org/10.1073/pnas.1707186114.
74. Wegener C, Veenstra JA. Chemical identity, function and regulation of enteroendocrine peptides in insects. Curr Opin Insect Sci. 2015;11:8-13. https://doi.org/10.1016/j.cois.2015.07.003.

75. Caccia S, Casartelli M, Tettamanti G. The amazing complexity of insect midgut cells: types, peculiarities, and functions. Cell Tissue Res. 2019;377(3): 505-25. https://doi.org/10.1007/s00441-019-03076-w.

76. Wu K, Li S, Wang J, Ni Y, Huang W, Liu Q, et al. Peptide hormones in the insect midgut. Front Physiol. 2020;11:191. https://doi.org/10.3389/fphys.2020. 00191.

77. Panz M, Vitos-Faleato J, Jendretzki A, Heinisch JJ, Paululat A, Meyer H. A novel role for the non-catalytic intracellular domain of neprilysins in muscle physiology. Biol Cell. 2012;104(9):553-68. https://doi.org/10.1111/boc.2011 00069.

78. Klein DC. Arylalkylamine N-acetyltransferase: "the Timezyme". J Biol Chem. 2007:282(7):4233-7. https://doi.org/10.1074/jbc.R600036200.

79. Hiragaki S, Suzuki T, Mohamed AA, Takeda M. Structures and functions of insect arylalkylamine $\mathrm{N}$-acetyltransferase (iaaNAT); a key enzyme for physiological and behavioral switch in arthropods. Front Physiol. 2015;6:113. https://doi.org/10.3389/fphys.2015.00113.

80. Amherd R, Hintermann E, Walz D, Affolter M, Meyer UA. Purification, cloning, and characterization of a second arylalkylamine $\mathrm{N}$-acetyltransferase from Drosophila melanogaster. DNA Cell Biol. 2000;19(11):697-705. https://doi. org/10.1089/10445490050199081.

81. Brodbeck D, Amherd R, Callaerts P, Hintermann E, Meyer UA, Affolter M. Molecular and biochemical characterization of the aaNAT1 (Dat) locus in Drosophila melanogaster: differential expression of two gene products. DNA Cell Biol. 1998;17(7):621-33. https://doi.org/10.1089/dna.1998.17.621.

82. Solari P, Rivelli N, De Rose F, Picciau L, Murru L, Stoffolano JG Jr, et al. Opposite effects of 5-HT/AKH and octopamine on the crop contractions in adult Drosophila melanogaster: Evidence of a double brain-gut serotonergic circuitry. PLOS ONE. 2017;12(3):e0174172. https://doi.org/10.1371/journal. pone.0174172.

83. Zhang B, Gong J, Zhang W, Xiao R, Liu J, Xu XZS. Brain-gut communications via distinct neuroendocrine signals bidirectionally regulate longevity in $C$. elegans. Genes Dev. 2018;32(3-4):258-70. https://doi.org/10.1101/gad.30962 5.117

84. Giltner CL, Nguyen Y, Burrows LL. Type IV pilin proteins: versatile molecular modules. Microbiol Mol Biol Rev. 2012;76(4):740-72. https://doi.org/10.1128/ MMBR.00035-12

85. Kazmierczak BI, Schniederberend M, Jain R. Cross-regulation of Pseudomonas motility systems: the intimate relationship between flagella, pili and virulence. Curr Opin Microbiol. 2015;28:78-82. https://doi.org/10.1016/j.mib.2 015.07.017.

86. Andrade M, Wang N. The Tad Pilus apparatus of 'Candidatus Liberibacter asiaticus' and its regulation by VisNR. Mol Plant Microbe Interact. 2019;32(9): 1175-87. https://doi.org/10.1094/MPMI-02-19-0052-R.

87. Andrade $\mathrm{MO}$, Pang $\mathrm{Z}$, Achor DS, Wang $\mathrm{H}$, Yao $\mathrm{T}$, Singer $\mathrm{BH}$, et al. The flagella of 'Candidatus Liberibacter asiaticus' and its movement in planta. Mol Plant Pathol. 2020:21:109-23. https://doi.org/10.1111/mpp.12884.

88. Bardy SL, Ng SYM, Jarrell KF. Prokaryotic motility structures. Microbiology. 2003:149:295-304. https://doi.org/10.1099/mic.0.25948-0.

89. Liu R, Ochman $\mathrm{H}$. Origins of flagellar gene operons and secondary flagellar systems. J Bacteriol. 2007;189(19):7098-104. https://doi.org/10.1128/JB. 00643-07.

90. Kostygov AY, Frolov AO, Malysheva MN, Ganyukova Al, Chistyakova LV, Tashyreva D, et al. Vickermania gen. nov., trypanosomatids that use two joined flagella to resist midgut peristaltic flow within the fly host. BMC Biol. 2020;18(1):187. 10.1186/s12915-020-00916-y.

91. Moens S, Vanderleyden J. Functions of bacterial flagella. Crit Rev Microbiol. 1996;22(2):67-100. https://doi.org/10.3109/10408419609106456.

92. Macnab RM. How bacteria assemble flagella. Annu Rev Microbiol. 2003;57: 77-100. https://doi.org/10.1146/annurev.micro.57.030502.090832.

93. Duan Q, Zhou M, Zhu L, Zhu G. Flagella and bacterial pathogenicity. J Basic Microbiol. 2013;53(1):1-8. https://doi.org/10.1002/jobm.201100335.

94. Matilla MA, Krell T. The effect of bacterial chemotaxis on host infection and pathogenicity. FEMS Microbiol Rev. 2018;42(1). https://doi.org/10.1093/ femsre/fux052.

95. Fong JNC, Yildiz FH. Biofilm matrix proteins. Microbiol Spectr. 2015;3(2):10. 1128/microbiolspec. MB-0004-2014. 10.1128/microbiolspec. MB-0004-2014.

96. Eshwar AK, Guldimann C, Oevermann A, Tasara T. Cold-shock domain family proteins (Csps) are involved in regulation of virulence, cellular aggregation, 
and flagella-based motility in Listeria monocytogenes. Front Cell Infect Microbiol. 2017;7:453. https://doi.org/10.3389/fcimb.2017.00453.

97. Ray S, Da Costa R, Thakur S, Nandi D. Salmonella Typhimurium encoded cold shock protein $\mathrm{E}$ is essential for motility and biofilm formation. Microbiology. 2020;166(5):460-73. https://doi.org/10.1099/mic.0.000900.

98. Nardi JB, Miller LA, Bee CM. Interfaces between microbes and membranes of host epithelial cells in hemipteran midguts. J Morphol. 2019;280(7):104660. https://doi.org/10.1002/jmor.21000.

99. Ghanim M, Achor D, Ghosh S, Kontsedalov S, Lebedev G, Levy A. 'Candidatus Liberibacter asiaticus' accumulates inside endoplasmic reticulum associated vacuoles in the gut cells of Diaphorina citri. Sci Rep. 2017;7(1): 16945. 10.1038/s41598-017-16095-W.

100. Ramsey JS, Johnson RS, Hoki JS, Kruse A, Mahoney J, Hilf ME, et al. Metabolic interplay between the asian citrus psyllid and its Profftella symbiont: an Achilles' heel of the citrus greening insect vector. PLOS ONE. 2015;10(11):e0140826. https://doi.org/10.1371/journal.pone.0140826.

101. Hansson GC. Role of mucus layers in gut infection and inflammation. Curr Opin Microbiol. 2012;15(1):57-62. https://doi.org/10.1016/j.mib.2011.11.002.

102. Silva CP, Silva JR, Vasconcelos FF, Petretski MD, Damatta RA, Ribeiro AF, et al. Occurrence of midgut perimicrovillar membranes in paraneopteran insect orders with comments on their function and evolutionary significance. Arthropod Struct Dev. 2004;33(2):139-48. https://doi.org/10.101 6/j.asd.2003.12.002

103. Kuraishi T, Binggeli O, Opota O, Buchon N, Lemaitre B. Genetic evidence for a protective role of the peritrophic matrix against intestinal bacterial infection in Drosophila melanogaster. Proc Natl Acad Sci U S A. 2011;108(38): 15966-71. https://doi.org/10.1073/pnas.1105994108.

104. Paone P, Cani PD. Mucus barrier, mucins and gut microbiota: the expected slimy partners? Gut. 2020;69(12):2232-43. https://doi.org/10.1136/gutjnl-202 0-322260.

105. Schroeder BO. Fight them or feed them: how the intestinal mucus layer manages the gut microbiota. Gastroenterol Rep (Oxf). 2019;7(1):3-12. https://doi.org/10.1093/gastro/goy052.

106. Wu P, Sun P, Nie K, Zhu Y, Shi M, Xiao C, et al. A gut commensal bacterium promotes mosquito permissiveness to arboviruses. Cell Host Microbe. 2019; 25(1):101-112.e5. 10.1016/j.chom.2018.11.004.

107. Ramsey JS, Chavez JD, Johnson R, Hosseinzadeh S, Mahoney JE, Mohr JP, et al. Protein interaction networks at the host-microbe interface in Diaphorina citri, the insect vector of the citrus greening pathogen. R Soc Open Sci. 2017:4(2):160545. https://doi.org/10.1098/rsos.160545.

108. Dohrman A, Miyata S, Gallup M, Li JD, Chapelin C, Coste A, et al. Mucin gene (MUC 2 and MUC 5AC) upregulation by Gram-positive and Gramnegative bacteria. Biochim Biophys Acta. 1998;1406(3):251-9. https://doi. org/10.1016/s0925-4439(98)00010-6.

109. Quintana-Hayashi MP, Mahu M, De Pauw N, Boyen F, Pasmans F, Martel A, et al. The levels of Brachyspira hyodysenteriae binding to porcine colonic mucins differ between individuals, and binding is increased to mucins from infected pigs with de novo MUC5AC synthesis. Infect Immun. 2015;83(4): 1610-9. https://doi.org/10.1128/IAl.03073-14

110. Matsuoka Y, Li X, Bennett V. Adducin: structure, function and regulation. Cell Mol Life Sci. 2000;57(6):884-95. https://doi.org/10.1007/PL00000731.

111. Hopkins AM, Walsh SV, Verkade $P$, Boquet $P$, Nusrat A. Constitutive activation of Rho proteins by CNF-1 influences tight junction structure and epithelial barrier function. J Cell Sci. 2003;116:725-42. https://doi.org/10.1242/jcs.00300.

112. Faggioni $R$, Feingold KR, Grunfeld $C$. Leptin regulation of the immune response and the immunodeficiency of malnutrition. FASEB J. 2001;15(14): 2565-71. https://doi.org/10.1096/fj.01-0431 rev.

113. Guo X, Roberts MR, Becker SM, Podd B, Zhang Y, Chua SC Jr, et al. Leptin signaling in intestinal epithelium mediates resistance to enteric infection by Entamoeba histolytica. Mucosal Immunol. 2011;4(3):294-303. https://doi. org/10.1038/mi.2010.76.

114. Mackey-Lawrence NM, Petri WA Jr. Leptin and mucosal immunity. Mucosal Immunol. 2012;5(5):472-9. https://doi.org/10.1038/mi.2012.40.

115. Stenbeck G. Soluble NSF-attachment proteins. Int J Biochem Cell Biol. 1998; 30(5):573-7. https://doi.org/10.1016/s1357-2725(97)00064-2.

116. Rothman JE. Mechanisms of intracellular protein transport. Nature. 1994; 372(6501):55-63. https://doi.org/10.1038/372055a0.

117. Lakhssassi N, Liu S, Bekal S, Zhou Z, Colantonio V, Lambert K, et al. Characterization of the soluble NSF attachment protein gene family identifies two members involved in additive resistance to a plant pathogen. Sci Rep. 2017;7:45226. https://doi.org/10.1038/srep45226.
118. Cerveny L, Straskova A, Dankova V, Hartlova A, Ceckova M, Staud F, et al. Tetratricopeptide repeat motifs in the world of bacterial pathogens: role in virulence mechanisms. Infect Immun. 2013;81(3):629-35. https://doi.org/1 $0.1128 /$ IAl.01035-12

119. Tjota M, Lee SK, Wu J, Williams JA, Khanna MR, Thomas GH. Annexin B9 binds to $\beta(H)$-spectrin and is required for multivesicular body function in Drosophila. J Cell Sci. 2011;124:2914-26. https://doi.org/10.1242/jcs.078667.

120. Norville IH, Breitbach K, Eske-Pogodda K, Harmer NJ, Sarkar-Tyson M, Titball RW, et al. A novel FK-506-binding-like protein that lacks peptidyl-prolyl isomerase activity is involved in intracellular infection and in vivo virulence of Burkholderia pseudomallei. Microbiology. 2011;157:2629-38. https://doi. org/10.1099/mic.0.049163-0.

121. Pandey S, Tripathi D, Khubaib M, Kumar A, Sheikh JA, Sumanlatha G, et al. Mycobacterium tuberculosis peptidyl-prolyl isomerases are immunogenic, alter cytokine profile and aid in intracellular survival. Front Cell Infect Microbiol. 2017;7:38. https://doi.org/10.3389/fcimb.2017.00038.

122. Rasch J, Ünal CM, Klages A, Karsli Ü, Heinsohn N, Brouwer RMHJ, et al. Peptidyl-prolyl-cis/trans-isomerases Mip and PpiB of Legionella pneumophila contribute to surface translocation, growth at suboptimal temperature, and infection. Infect Immun. 2018;87(1):e00939-17. https://doi.org/10.1128/IAI. 00939-17.

123. Helbig JH, König B, Knospe H, Bubert B, Yu C, Lück CP, et al. The PPlase active site of Legionella pneumophila Mip protein is involved in the infection of eukaryotic host cells. Biol Chem. 2003;384(1):125-37. 12674506. https:// doi.org/10.1515/BC.2003.013.

124. Celli J, Gorvel JP. Organelle robbery: Brucella interactions with the endoplasmic reticulum. Curr Opin Microbiol. 2004;7(1):93-7. https://doi.org/1 0.1016/j.mib.2003.11.001.

125. Robinson CG, Roy CR. Attachment and fusion of endoplasmic reticulum with vacuoles containing Legionella pneumophila. Cell Microbiol. 2006;8(5): 793-805. https://doi.org/10.1111/j.1462-5822.2005.00666.x.

126. Friedman JR, Dibenedetto JR, West $M$, Rowland AA, Voeltz GK. Endoplasmic reticulum-endosome contact increases as endosomes traffic and mature. Mol Biol Cell. 2013;24(7):1030-40. https://doi.org/10.1091/mbc.E12-10-0733.

127. Granger E, McNee G, Allan V, Woodman P. The role of the cytoskeleton and molecular motors in endosomal dynamics. Semin Cell Dev Biol. 2014; 31(100):20-9. https://doi.org/10.1016/j.semcdb.2014.04.011.

128. Witke $W$. The role of profilin complexes in cell motility and other cellular processes. Trends Cell Biol. 2004;14(8):461-9. https://doi.org/10.1016/j.tcb.2 004.07.003.

129. Kocks C. Intracellular motility. Profilin puts pathogens on the actin drive. Curr Biol. 1994;4(5):465-8. https://doi.org/10.1016/s0960-9822(00)00105-6.

130. Holzbaur EL, Vallee RB. Dyneins: molecular structure and cellular function. Annu Rev Cell Biol. 1994;10:339-72. https://doi.org/10.1146/annurev.cb.10.11 0194.002011.

131. Porter ME. Axonemal dyneins: assembly, organization, and regulation. Curr Opin Cell Biol. 1996;8(1):10-7. https://doi.org/10.1016/s0955-0674 (96)80042-1

132. Hirokawa N. Kinesin and dynein superfamily proteins and the mechanism of organelle transport. Science. 1998;279(5350):519-26. https://doi.org/10.1126/ science.279.5350.519.

133. Thapa SP, De Francesco A, Trinh J, Gurung FB, Pang Z, Vidalakis G, et al. Genome-wide analyses of Liberibacter species provides insights into evolution, phylogenetic relationships, and virulence factors. Mol Plant Pathol. 2020;21(5):716-31. https://doi.org/10.1111/mpp.12925.

134. Prasad S, Xu J, Zhang Y, Wang N. SEC-Translocon dependent extracytoplasmic proteins of Candidatus Liberibacter asiaticus. Front Microbiol. 2016;7:1989. https://doi.org/10.3389/fmicb.2016.01989.

135. Wang N, Pierson EA, Setubal JC, Xu J, Levy JG, Zhang Y, et al. The Candidatus Liberibacter-host interface: insights into pathogenesis mechanisms and disease control. Annu Rev Phytopathol. 2017:55:451-82. https://doi.org/10.1146/annurev-phyto-080516-035513.

136. Andrade M, Li JY, Wang N. Candidatus Liberibacter asiaticus: virulence traits and control strategies. Trop Plant Pathol. 2020b;45:285-97. https://doi.org/1 0.1007/s40858-020-00341-0.

137. Green ER, Mecsas J. Bacterial secretion systems: an overview. Microbiol Spectr. 2016;4(1):10.1128/microbiolspec. VMBF-0012-2015. 10.1128/ microbiolspec. VMBF-0012-2015.

138. McFarland L, Francetić O, Kumamoto CA. A mutation of Escherichia coli SecA protein that partially compensates for the absence of SecB. J Bacteriol. 1993;175(8):2255-62. https://doi.org/10.1128/jb.175.8.2255-2262.1993. 
139. Cicero JM, Fisher TW, Qureshi JA, Stansly PA, Brown JK. Colonization and intrusive invasion of potato psyllid by 'Candidatus Liberibacter solanacearum'. Phytopathology. 2017;107(1):36-49. https://doi.org/10.1094/ PHYTO-03-16-0149-R

140. Galluzzi L, Vitale I, Aaronson SA, Abrams JM, Adam D, Agostinis P, et al. Molecular mechanisms of cell death: recommendations of the Nomenclature Committee on Cell Death 2018. Cell Death Differ. 2018;25(3): 486-541. https://doi.org/10.1038/s41418-017-0012-4.

141. Vande Velde C, Cizeau J, Dubik D, Alimonti J, Brown T, Israels S, et al. BNIP3 and genetic control of necrosis-like cell death through the mitochondrial permeability transition pore. Mol Cell Biol. 2000;20(15):5454-68. https://doi. org/10.1128/mcb.20.15.5454-5468.2000.

142. Moy RH, Cherry S. Antimicrobial autophagy: a conserved innate immune response in Drosophila. J Innate Immun. 2013;5(5):444-55. https://doi.org/1 $0.1159 / 000350326$

143. Münch D, Amdam GV, Wolschin F. Ageing in a eusocial insect: molecular and physiological characteristics of life span plasticity in the honey bee. Funct Ecol. 2008;22(3):407-21. https://doi.org/10.1111/j.1365-2435.2008.0141 9.x.

144. Ito T, Igaki T. Dissecting cellular senescence and SASP in Drosophila. Inflamm Regen. 2016;36:25. https://doi.org/10.1186/s41232-016-0031-4

145. Zalewska M, Kochman A, Estève JP, Lopez F, Chaoui K, et al. Juvenile hormone binding protein traffic - Interaction with ATP synthase and lipid transfer proteins. Biochim Biophys Acta. 2009;1788(9):1695-705. https://doi. org/10.1016/j.bbamem.2009.04.022.

146. Rahman MM, Franch-Marro X, Maestro JL, Martin D, Casali A. Local juvenile hormone activity regulates gut homeostasis and tumor growth in adult Drosophila. Sci Rep. 2017;7(1):11677. https://doi.org/10.1038/s41598-017-111 99-9.

147. Bajgar A, Jindra M, Dolezel D. Autonomous regulation of the insect gut by circadian genes acting downstream of juvenile hormone signaling. Proc Natl Acad Sci U S A. 2013;110(11):4416-21. https://doi.org/10.1073/pnas.121 7060110.

148. Hughes KL, Abshire ET, Goldstrohm AC. Regulatory roles of vertebrate nocturnin: insights and remaining mysteries. RNA Biol. 2018;15(10):1255-67. https://doi.org/10.1080/15476286.2018.1526541.

149. Estrella MA, Du J, Chen L, Rath S, Prangley E, et al. The metabolites NADP+ and NADPH are the targets of the circadian protein nocturnin (Curled). Nat Commun. 2019;10(1):2367. https://doi.org/10.1038/s41467-019-10125-z.

150. Laothamatas I, Gao P, Wickramaratne A, Quintanilla CG, Dino A, et al. Spatiotemporal regulation of $\mathrm{NADP}(\mathrm{H})$ phosphatase nocturnin and its role in oxidative stress response. Proc Natl Acad Sci U S A. 2020;117(2):993-9. https://doi.org/10.1073/pnas.1913712117.

151. Redder P, Hausmann S, Khemici V, Yasrebi H, Linder P. Bacterial versatility requires DEAD-box RNA helicases. FEMS Microbiol Rev. 2015;39(3):392-412 https://doi.org/10.1093/femsre/fuv011.

152. Agashe VR, Guha S, Chang HC, Genevaux P, Hayer-Hartl M, Stemp M, et al. Function of trigger factor and DnaK in multidomain protein folding: increase in yield at the expense of folding speed. Cell. 2004;117(2):199-209. https://doi.org/10.1016/s0092-8674(04)00299-5.

153. Merz F, Hoffmann A, Rutkowska A, Zachmann-Brand B, Bukau B, Deuerling E. The C-terminal domain of Escherichia coli trigger factor represents the central module of its chaperone activity. J Biol Chem. 2006;281(42):3196371. https://doi.org/10.1074/jbc.M605164200.

154. Carr S, Penfold CN, Bamfold V, James R, Hemmings AM. The structure of TolB, an essential component of the tol-dependent translocation system and its protein-protein interaction with the translocation domain of colicin E9. Structure. 2000;8:57-66. https://doi.org/10.1016/S0969-2126(00)00079-4.

155. Loftus SR, Walker D, Maté MJ, Bonsor DA, James R, Moore GR, et al. Competitive recruitment of the periplasmic translocation portal TolB by a natively disordered domain of colicin E9. Proc Natl Acad Sci U S A. 2006; 103(33):12353-8. https://doi.org/10.1073/pnas.0603433103.

156. Santos CA, Janissen R, Toledo MA, Beloti LL, Azzoni AR, et al. Characterization of the TolB-Pal trans-envelope complex from Xylella fastidiosa reveals a dynamic and coordinated protein expression profile during the biofilm development process. Biochim Biophys Acta. 1854;2015 1372-81. https://doi.org/10.1016/j.bbapap.2015.05.018.

157. Theodorou MC, Theodorou EC, Kyriakidis DA. Involvement of AtoSC twocomponent system in Escherichia coli flagellar regulon. Amino Acids. 2012; 43(2):833-44. https://doi.org/10.1007/s00726-011-1140-7.
158. Beinert H. A tribute to sulfur. Eur J Biochem. 2000;267:5657-64. https://doi org/10.1046/j.1432-1327.2000.01637.x

159. Layer G, Gaddam SA, Ayala-Castro CN, Ollagnier-de Choudens S, Lascoux D, et al. SufE transfers sulfur from SufS to SufB for iron-sulfur cluster assembly. J Biol Chem. 2007;282(18):13342-50. https://doi.org/10.1074/jbc.M6085552 00.

160. Roche B, Aussel L, Ezraty B, Mandin P, Py B, Barras F. Iron/sulfur proteins biogenesis in prokaryotes: formation, regulation and diversity. Biochim Biophys Acta. 2013;1827(3):455-69. https://doi.org/10.1016/j.bbabio.2012.12. 010.

161. Lu J, Holmgren A. The thioredoxin antioxidant system. Free Radic Biol Med. 2014;66:75-87. https://doi.org/10.1016/j.freeradbiomed.2013.07.036.

162. Wang C, Chen Y, Zhou H, Li X, Tan Z. Adaptation mechanisms of Rhodococcus sp. CNS16 under different temperature gradients: Physiological and transcriptome. Chemosphere. 2020;238:124571. https://doi.org/10.1016/ j.chemosphere.2019.124571.

163. Lamarche MG, Wanner BL, Crépin S, Harel J. The phosphate regulon and bacterial virulence: a regulatory network connecting phosphate homeostasis and pathogenesis. FEMS Microbiol Rev. 2008;32(3):461-73. https://doi.org/10.1111/j.1574-6976.2008.00101.x.

164. Santos-Beneit $F$. The Pho regulon: a huge regulatory network in bacteria. Front Microbiol. 2015;6:402. https://doi.org/10.3389/fmicb.2015.00402.

165. Sohlenkamp C, López-Lara IM, Geiger O. Biosynthesis of phosphatidylcholine in bacteria. Prog Lipid Res. 2003;42(2):115-62. https:// doi.org/10.1016/s0163-7827(02)00050-4.

166. Dupont L, Garcia I, Poggi MC, Alloing G, Mandon K, Le Rudulier D. The Sinorhizobium meliloti $A B C$ transporter Cho is highly specific for choline and expressed in bacteroids from Medicago sativa nodules. J Bacteriol. 2004; 186(18):5988-96. https://doi.org/10.1128/JB.186.18.5988-5996.2004.

167. Li W, Cong Q, Pei J, Kinch LN, Grishin NV. The ABC transporters in Candidatus Liberibacter asiaticus. Proteins. 2012;80(11):2614-28. https://doi. org/10.1002/prot.24147.

168. Manjunath KL, Halbert SE, Ramadugu C, Webb S, Lee RF. Detection of 'Candidatus Liberibacter asiaticus' in Diaphorina citri and its importance in the management of citrus huanglongbing in Florida. Phytopathology. 2008; 98(4):387-96. https://doi.org/10.1094/PHYTO-98-4-0387.

169. Li W, Hartung JS, Levy L. Quantitative real-time PCR for detection and identification of Candidatus Liberibacter species associated with citrus huanglongbing. J Microbiol Methods. 2006;66(1):104-15. https://doi.org/10.1 016/j.mimet.2005.10.018.

170. Andrews S. FastQC: a quality control tool for high throughput sequence data [software]. 2010 [cited 2021 Feb 26]. Available from: http://www. bioinformatics.babraham.ac.uk/projects/fastqc.

171. Bolger AM, Lohse M, Usadel B. Trimmomatic: a flexible trimmer for Illumina sequence data. Bioinformatics. 2014;30(15):2114-20. https://doi.org/10.1093/ bioinformatics/btu170.

172. Haas BJ, Papanicolaou A, Yassour M, Grabherr M, Blood PD, Bowden J, et al. De novo transcript sequence reconstruction from RNA-seq using the Trinity platform for reference generation and analysis. Nat Protoc. 2013;8(8):1494512. https://doi.org/10.1038/nprot.2013.084.

173. Conesa A, Götz S, García-Gómez JM, Terol J, Talón M, Robles M. Blast2GO: a universal tool for annotation, visualization and analysis in functional genomics research. Bioinformatics. 2005;21(18):3674-6. https://doi.org/10.1 093/bioinformatics/bti610.

174. Huerta-Cepas J, Forslund K, Coelho LP, Szklarczyk D, Jensen LJ, von Mering $C$, et al. Fast genome-wide functional annotation through orthology assignment by eggNOG-Mapper. Mol Biol Evol. 2017;34(8):2115-22. https:// doi.org/10.1093/molbev/msx148.

175. Kanehisa M, Goto S. KEGG: Kyoto encyclopedia of genes and genomes. Nucleic Acids Res. 2000;28(1):27-30. https://doi.org/10.1093/nar/28.1.27.

176. Götz S, García-Gómez JM, Terol J, Williams TD, Nagaraj SH, Nueda MJ, et al. High-throughput functional annotation and data mining with the Blast2GO suite. Nucleic Acids Res. 2008;36(10):3420-35. https://doi.org/10.1093/nar/ gkn176.

\section{Publisher's Note}

Springer Nature remains neutral with regard to jurisdictional claims in published maps and institutional affiliations. 